\title{
金属有机框架物催化有机反应综述
}

\author{
徐光利刚芳莉董涛生傅颖杜正银* \\ (西北师范大学化学化工学院 兰州 730070)
}

\begin{abstract}
摘要 金属有机框架物(MOFs)是近十多年发展起来的一类新型有机-无机杂化材料，由有机配体和无机金属单元构建 而成，一般具有多变的拓扑结构以及独特的物理化学性质. MOFs 具有特殊的孔洞框架结构, 材料种类繁多，比表面积 大，其孔穴的大小、形状及构成等可以通过选择不同配体和金属离子，或者改变合成策略加以调节. 构成 MOFs 的配体 可以是有机酸，也可以是有机碱，还可以是其他特殊的结构，除此之外在配体上也可以连接特殊官能团，制备具有特 殊功能的 MOFs 材料. 由于 MOFs 具有特殊而又多种多样的结构, 因此在功能材料、气体吸附、药物缓释、催化及有 机合成等方面有广泛的应用. 本文以形成 MOFs 的金属为分类就近十年来各种 MOFs 的制备及其在催化有机反应中的 应用进行了综述, 并对其未来研究加以展望.
\end{abstract}

关键词 金属有机框架物(MOFs); 制备; 多相催化; 有机合成; 应用

\section{Application of Metal Organic Frameworks in Catalytic Organic Reactions}

\author{
Xu, Guangli Gang, Fangli Dong, Taosheng Fu, Ying Du, Zhengyin* \\ (College of Chemistry and Chemical Engineering, Northwest Normal University, Lanzhou730070)
}

\begin{abstract}
Metal organic frameworks (MOFs) as a new type of organic/inorganic hybrid materials have attracted great focus of scientists in almost twenty years. It is composed of organic ligands and inorganic metal units and generally has a varied topological structure and unique physical/chemical properties. Due to its porous frame structure, large specific surface area and great variety, MOFs are widely used in functional materials, gas adsorption, drug sustained release, catalysis and organic synthesis. It can choose different ligands and metal ions, or change the strategy of synthesis to adjust the size, shape and structure of porous structure. The different ligands can determine the different structure and then affect the properties of MOFs. In this review, the preparation of particular functional MOFs structure and the application of various MOFs in organic synthesis and catalytic organic reactions are reviewed in detail. The summary and prospects about MOFs catalyzed organic reactions and the applications are proposed.
\end{abstract}

Keywords metal organic frameworks (MOFs); preparation; heterogeneous catalysis; organic synthesis; application

金属一有机框架材料(Metal-Organic Frameworks, MOFs) 是一类有机-无机杂化材料, 由有机配体和无机 金属通过自组装形成的以金属为节点的网状结构的材 料, 一般具有多变的拓扑结构以及物理化学性质. 由于 是网状骨架结构, 所以具有很大的比表面积, 其 Langmuir 比表面积可达到 $4500 \mathrm{~m}^{2} \cdot \mathrm{g}^{-1}$ 甚至更大 ${ }^{[1]}$. MOFs 通过与不同的金属自组装, 可形成不同的孔洞均 一的可控结构, 携带的金属催化剂可对反应物形成像分 子篮一样的选择性催化 ${ }^{[2 ~ 4]}$; 另外, 金属裸露在空隙之
间，使其催化性能大大提高，在催化有机合成中的应用 也十分广泛; 此外, 孔洞结构为其提供了吸附气体的功 能 $^{[5 \sim 8]}$. 基于诸多功能, 近几年来, MOFs 的合成与应用 已成为国内外化学科研工作者研究的热点 ${ }^{[9 \sim 17]}$, 也有一 些关于 MOFs 的制备与应用的综述 ${ }^{[18 \sim 21]}$, 但当前关于 MOFs 材料作为催化剂在有机合成中的应用的综述并不 多见. 本文将对各种 MOFs 材料作为多相催化剂在催化 有机合成反应中的应用作一详细的综述, 并对其应用前 景加以展望.

*E-mail: Clinton_du@126.com

Received January 20, 2016; revised March 2, 2016; published online March 28, 2016.

Project supported by the National Natural Science Foundation of China (No. 21262028) and the Natural Science Foundation of Gansu Province (No. 1208RJZA140).

国家自然科学基金(No. 21262028)及甘肃省自然科学基金(No. 1208RJZA140)资助项目. 


\section{$1 \mathrm{Cu}-M O F s$ 在催化有机反应中的应用}

2013 年 Anbu 课题组 ${ }^{[22]}$ 用电化学方法, 以纯度为 $99.9 \%$ 的铜电极和均苯三甲酸 $\left(\mathrm{H}_{3} \mathrm{BTC}\right)$ 为原料, 合成了 $\mathrm{Cu}_{3}(\mathrm{BTC})_{2}$. 该催化剂中, $\mathrm{Cu}$ 是以 $\mathrm{Cu}^{2+}$ 的形式存在于骨 架的节点上, $\mathrm{H}_{3} \mathrm{BTC}$ 提供了支撑骨架. 作者以 $\mathrm{Cu}_{3}(\mathrm{BTC})_{2}$ 为催化剂, $\mathrm{NaBH}_{4}$ 为还原剂来催化硝基芳烃 的还原(Scheme 1). 该方法避免了使用氢气, 简化了反 应条件, 在 $50{ }^{\circ} \mathrm{C}$ 下就得到了还原产物, 产率在 $95 \%$ 以 上. 与传统的金属加无机酸和氢气还原体系相比, 不仅 条件简单、收率高，而且环保安全.

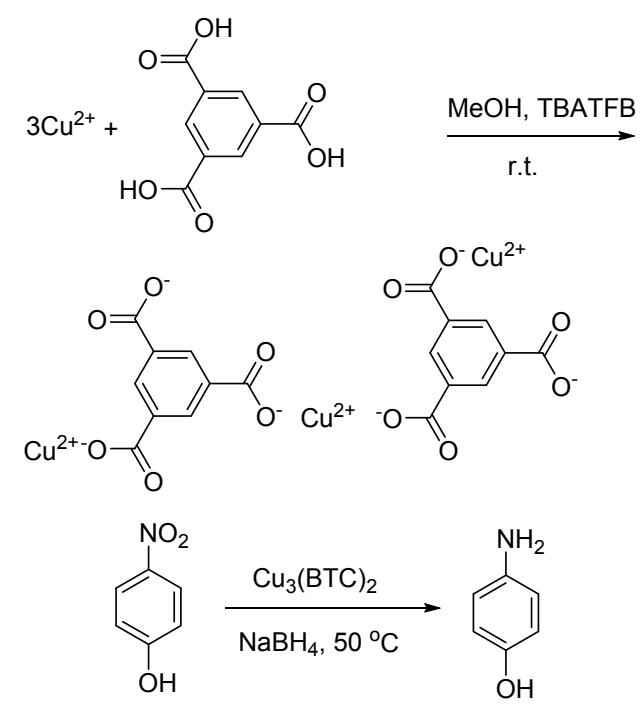

图式 $1 \mathrm{Cu}_{3}(\mathrm{BTC})_{2}-\mathrm{MOF}$ 的制备及应用

Scheme 1 Preparation and application of $\mathrm{Cu}_{3}(\mathrm{BTC})_{2}-\mathrm{MOF}$

环氧化合物的开环反应是有机化学研究的主要课 题之一. 通过开环反应, 可以制备多种带有官能团的化 合物, 而传统的开环反应一般需要 $\mathrm{Ag} 、 \mathrm{Pd}$ 等贵金属作 为催化剂, 不仅成本高, 而且产率一般较低. 2009 年 Baiker 等 ${ }^{[23]}$ 以 $\mathrm{Cu}\left(\mathrm{BF}_{4}\right)_{2} \cdot \mathrm{H}_{2} \mathrm{O}$ 和联二吡啶(BPY)为原料, 合成了 $\left[\mathrm{Cu}(\mathrm{BPY})_{2}\left(\mathrm{H}_{2} \mathrm{O}\right)_{2}\left(\mathrm{BF}_{4}\right)_{2}\right]$ 型 $\mathrm{Cu}-\mathrm{MOF}$ (Scheme 2), 其中 $\mathrm{Cu}^{2+}$ 提供金属配位点, 4,4'-联吡定来形成三维互穿 骨架. 在甲醇的参与下该 $\mathrm{Cu}-\mathrm{MOF}$ 原来的骨架会发生重 建, 失去 $\mathrm{BF}_{4}{ }^{-}$和一分子水, 具有亲和性的吡啶基团与 $\mathrm{Cu}^{2+}$ 相连, 形成具有高活性的三维骨架. 研究发现以甲 醇为溶剂, 以该 $\mathrm{Cu}-\mathrm{MOF}$ 为催化剂, 室温下反应 $2 \mathrm{~h}$, 取 代环氧乙烷就能发生醇解开环生成羟基化产物, 产率在 93\%以上.

$\mathrm{Cu}-\mathrm{MOF}$ 不仅可以催化环氧化合物的开环反应, 也 可以催化环氧化合物的合成反应. Baiker 课题组 ${ }^{[24]}$ 运用 逆向思维研究了 MOFs 催化烯烃的环氧化反应, 以 $\mathrm{Cu}\left(\mathrm{BF}_{4}\right)_{2} \bullet \mathrm{H}_{2} \mathrm{O}$ 和联二吡啶为原料, 合成了上述 $\mathrm{Cu}-\mathrm{MOF}$,

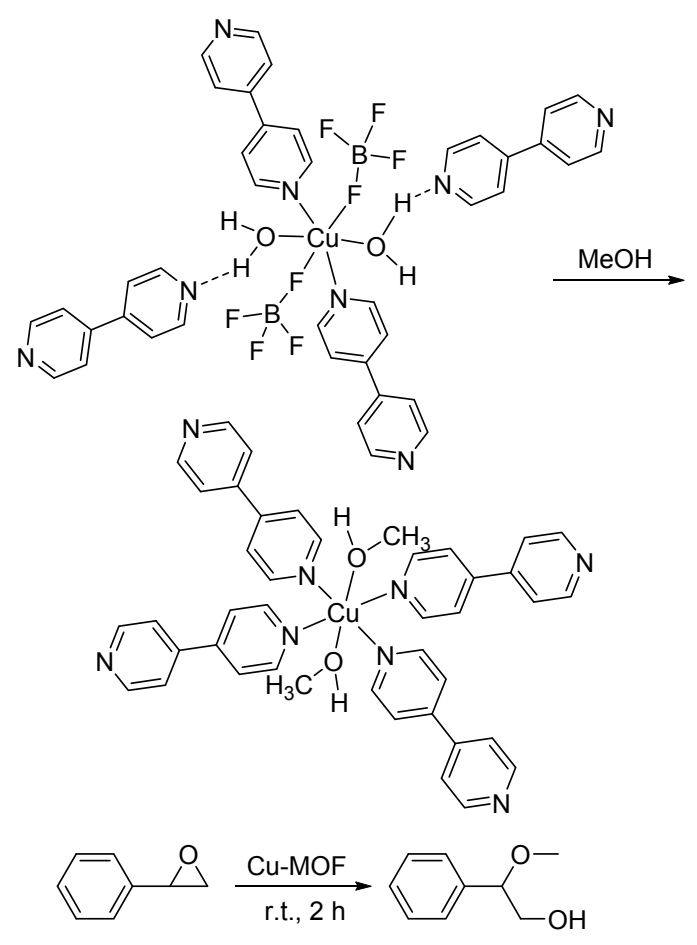

图式 $2 \mathrm{Cu}-\mathrm{MOF}$ 的活化和应用

Scheme 2 The activation and application of $\mathrm{Cu}-\mathrm{MOF}$

该催化剂以联二吡啶为支柱构建了 MOF 骨架, 以二价 铜离子为结点. 利用该 $\mathrm{Cu}-\mathrm{MOF}$ 作为催化剂, 成功实现 了环己烯烃的环氧化反应(Eq. 1), 在乙醇中 $45{ }^{\circ} \mathrm{C}$ 下反 应 $15 \mathrm{~h}$, 高产率的得到了环已烯环氧化合物, 反应条件 温和，操作简单易控制，更重要的是直接用廉价的铜作 为催化剂, 催化烯烃的氧化环化反应, 形成了一条通过 控制温度来实现由烯烃化合物制备环状化合物进而开 环的反应链. 同时以环氧化合物为原料进一步发生开环 反应生成带有其他官能团的化合物, 这对于合成带有特 殊官能团的化合物具有十分重要的意义.

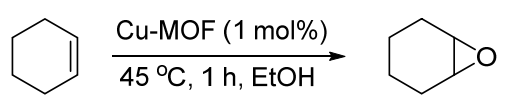

2015 年, Wang 课题组 ${ }^{[25]}$ 首先用十二烷酸和苯甲酸 与 $\mathrm{Cu}\left(\mathrm{NO}_{3}\right)_{2} \cdot 3 \mathrm{H}_{2} \mathrm{O}$ 在一定条件下反应, 然后加入 $\mathrm{H}_{3} \mathrm{BTC}$ 制备了两种纳米级的 $\mathrm{Cu}_{3}(\mathrm{BTC})_{2}$ 型 $\mathrm{MOFs}$ 催化剂 Cu-MOF-1 和 Cu-MOF-2, 平均粒径分别是 90 和 $390 \mathrm{~nm}$. 研究发现, Cu-MOF-1 和 Cu-MOF-2 都能高效地催化烯 烃的环氧化反应(Eq. 2). 该方法不仅适用于六元环烯烃 的环氧化, 还适用于八元环、十元环, 甚至桥环烯烃和 脂肪族烯烃的环氧化. 和 Baiker 课题组 ${ }^{[24]}$ 的 MOFs 催化 烯烃环氧化方法相比，该方法应用范围更广. 


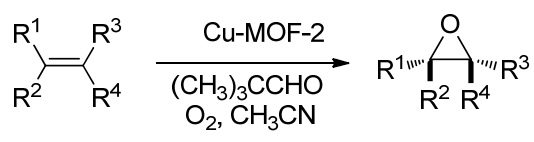

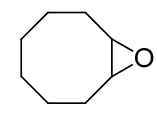

$99 \%$
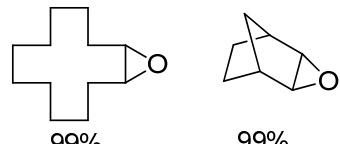

$99 \%$<smiles>c1ccc(C2OC2c2cccc3c2CCC2OC32)cc1</smiles>

$99 \%$
$80 \%$

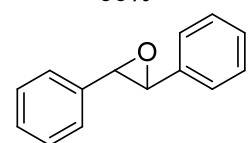

$99 \%$
$75 \%$

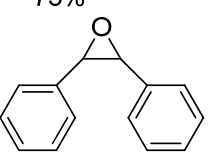

$63 \%$

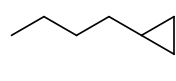

Cu-MOF-2 除了能催化烯烃的环氧化反应外, 还能 催化伯醇发生高选择性的氧化反应生成醛(Eq. 3), 但是 对仲醇却没有催化效果.
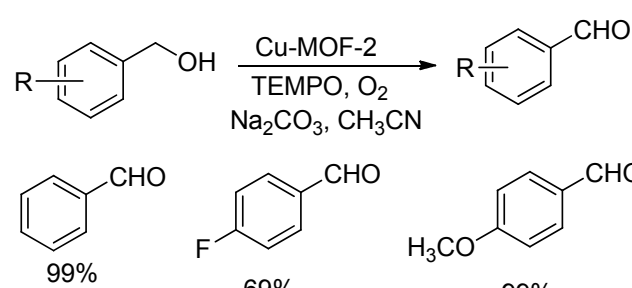<smiles>O=Cc1ccc(F)cc1</smiles><smiles>COc1ccc(C=O)cc1</smiles><smiles>CC(=O)c1ccccc1</smiles>

0<smiles>O=C(c1ccccc1)c1ccccc1</smiles>

0<smiles>O=C/C=C/c1ccccc1</smiles>

$99 \%$

$\mathrm{N}$-烷基化反应在有机反应、医药合成、材料科学等 领域有重要的应用, 尤其是含有 $\beta$-羰基酯的化合物作为 烷基化试剂的氮烷基化反应在有机合成中尤为重要. 2012 年, $\mathrm{Phan}$ 等 ${ }^{[26]}$ 用 $\mathrm{Cu}\left(\mathrm{NO}_{3}\right)_{2} \cdot 3 \mathrm{H}_{2} \mathrm{O}$ 和 $\mathrm{H}_{3} \mathrm{BTC}$ 为原料, 在 DMF-乙醇-水的混合溶剂中合成了 Cu-MOF199, 并 以此为催化剂, 以芐胺和丙烯酸乙酯为原料发生 $\mathrm{N}$ 杂迈 克尔加成反应, 生成含有 $\beta$-羊炭基酯结构的 $\mathrm{N}$-烷基化产 物(Eq. 4). 与传统的合成方法相比, 该方法采用了廉价 易得的 Cu-MOF 作为催化剂, 条件温和, 室温下不需要 添加剂就得到了 $\mathrm{N}$-烷基化产物. 通过改变芳胺与酯的 比例还可以控制产物的选择性. 当芳胺与酯的比例小于 $1: 2$ 时, 以 $\mathbf{A}$ 为主产物; 相反, 当比列大于 $1: 2$ 时, 以 B 为主产物.

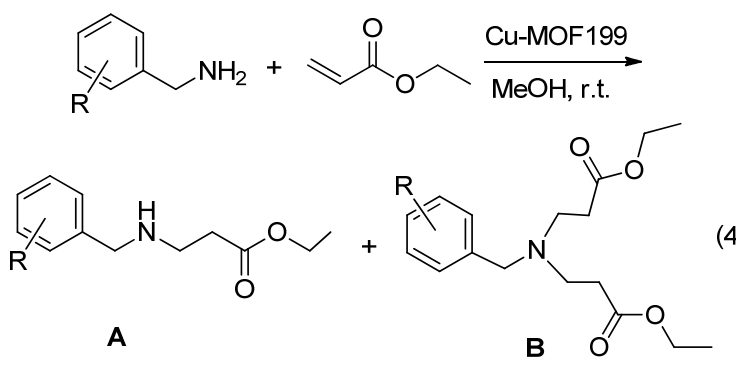

喹啉类化合物具有重要的生物活性, 并且在医药和 有机合成中有重要的应用. 喹啉衍生物一般采用斯克劳 普法合成, 但反应时间长, 产率相对较低 ${ }^{[27]} .2013$ 年, Phan 课题组 ${ }^{[28]}$ 用 $\mathrm{Cu}\left(\mathrm{NO}_{3}\right)_{2} \cdot 3 \mathrm{H}_{2} \mathrm{O}$ 和对苯二甲酸 $\left(\mathrm{H}_{2} \mathrm{BDC}\right)$ 为原料，用简便的方法合成了 $\mathrm{Cu}(\mathrm{BDC})$ 型 $\mathrm{MOF}$, 并以此 为催化剂, 通过 2-氨基茮醇和对位取代的苯乙酮合成了 2-取代的喹啉(Eq. 5). 结果发现，该方法条件温和，反 应时间短，只需 $40 \mathrm{~min}$ 即可反应完全，产率能达到 $92 \%$, 而且，苯乙酮上连接不同的取代基，产率都会在 $90 \%$ 以 上，是真正的原子经济性反应. 另外，在该反应中催化 剂可以重复利用，经过 8 次反应后产率仍然能达到 $85 \%$ 以上.
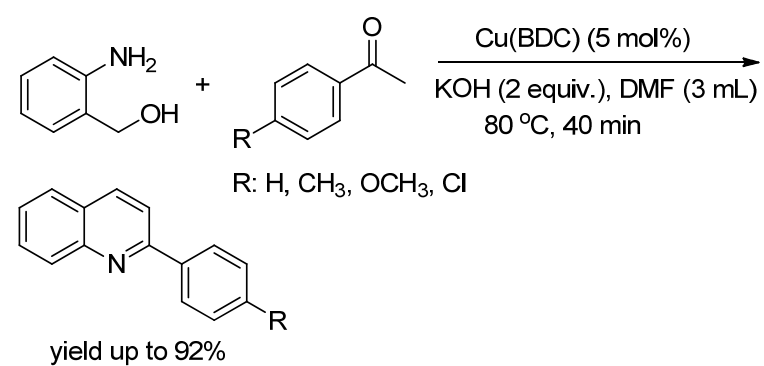

喹噁啉作为抗结核药物吡嗪酰胺的中间体，在有机 合成、药物合成中有重要的应用. 传统的方法是以二胺 和乙二醛为原料来合成的, 操作复杂, 产率较低. 2014 年, Phan 课题组 ${ }^{[29]}$ 经过探索发现 $\mathrm{Cu}(\mathrm{BDC})-\mathrm{MOF}$ 还能催 化生成喹噁啉. 以 $\mathrm{Cu}(\mathrm{BDC})-\mathrm{MOF}$ 为催化剂, 以 2-羊基 苯乙酮和邻苯二胺为原料, 甲苯为溶剂, 在 $100{ }^{\circ} \mathrm{C}$ 下、 空气氛围中合成了带有芳基的喹噁啉化合物，产率高达 95\% (Eq. 6). 这一方法的建立对于喹噁啉类化合物的高 效合成具有十分重要的意义.

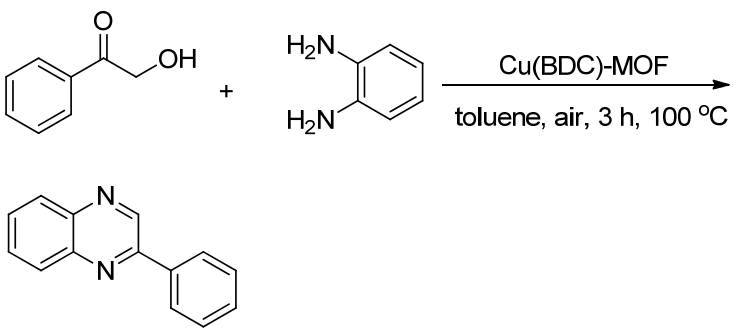

2014 年, Martínez 等 ${ }^{[30]}$ 用 $\mathrm{Cu}\left(\mathrm{NO}_{3}\right)_{2} \cdot 3 \mathrm{H}_{2} \mathrm{O}$ 和 2,5-二 羟基对苯二甲酸，采用高压反应法合成了 $\mathrm{Cu}-\mathrm{MOF} 74$. 与对苯二甲酸为骨架的 Cu-MOF74 相比, Cu-MOF74 骨 架带有活性的羟基官能团. 研究发现, 以 $\mathrm{Cu}-\mathrm{MOF} 74$ 为 催化剂在 $120{ }^{\circ} \mathrm{C}$ 下苯甲醚和酰氯很容易发生傅克酰基 化反应，生成苯环邻位或对位取代的酰基化产物(Eq. 7). 通过控制反应温度和物料比能实现对产物选择性的控 制，当温度从 $60{ }^{\circ} \mathrm{C}$ 升高到 $120{ }^{\circ} \mathrm{C}$ 时，对位产物的产率 从 $35 \%$ 逐渐升高到 $79 \%$; 当苯甲醚和酰氯的比例从 1 : 
1 提高到 5:1 时, 对位产物的产率从 55\%逐渐升高到 $98 \%$.

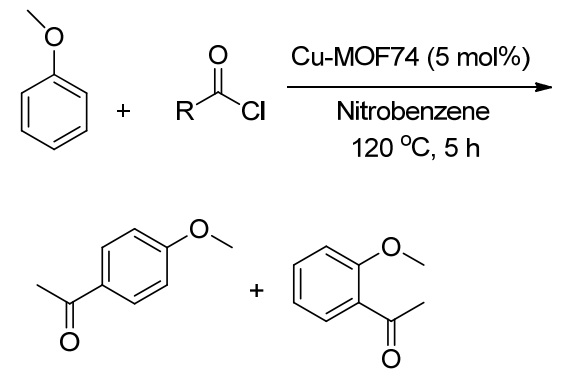

2013 年, Phan 课题组 ${ }^{[31]}$ 用 $\mathrm{Cu}\left(\mathrm{NO}_{3}\right)_{2} \cdot 3 \mathrm{H}_{2} \mathrm{O}$ 和 $\mathrm{H}_{3} \mathrm{BTC}$ 为原料, 以 $\mathrm{DMF}$ 为溶剂, 在一定条件下制备了 $\mathrm{Cu}-\mathrm{MOF} 199$. 以 $\mathrm{Cu}-\mathrm{MOF} 199$ 为催化剂, 以取代碘苯和 取代苯酚为原料, 成功实现了 Ullmann 偶联反应(Eq. 8), 通过实验发现，该催化剂重复利用 5 次以后该反应的产 物收率仍能保持在 $80 \%$.

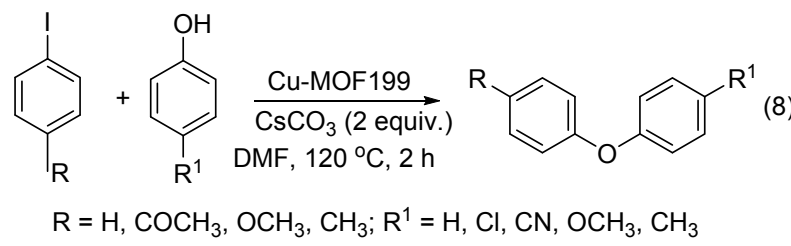

2013 年 $\mathrm{Zhao}$ 等 ${ }^{[32]}$ 用 $\mathrm{CuSO}_{4} \cdot 5 \mathrm{H}_{2} \mathrm{O}$ 和咪唑-1-乙酸为 原料, 制备了相应的 $\mathrm{Cu}-\mathrm{MOF}$ 复合物. 其后作者以此 $\mathrm{Cu}-\mathrm{MOF}$ 为催化剂, 以苯硼酸和咪唑为原料, 在室温下 反应 $5 \mathrm{~h}$, 得到了杂环的 $\mathrm{N}$-芳基化产物(Eq. 9). 研究结 果表明苯嗍酸上取代基的电子效应对该反应影响较大, 供电子基团有助于该反应的进行，反应产率高达 $92 \%$; 相反, 吸电子基团则对该反应有明显的阻碍作用, 反应 产率下降到 $71 \%$.

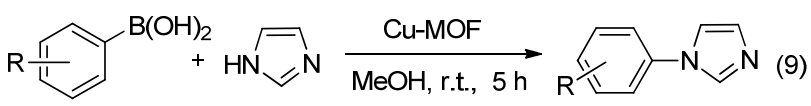<smiles>COc1ccc([18O]c2ccc([18OH])cc2)cc1</smiles>

三氮唑类化合物不仅在医药合成方面有重要的应 用, 而且在小分子合成、材料科学、纳米粒子和纳米载 体等方面有广泛应用. 2010 年, Corma 课题组 ${ }^{[33]}$ 用 $\mathrm{Cu}(\mathrm{OAc})_{2} \cdot \mathrm{H}_{2} \mathrm{O}$ 和 $\mathrm{H}_{3} \mathrm{BTC}$ 为原料, 在一定条件下制备了 相应的 $\mathrm{Cu}-\mathrm{MOF}$. 在该 MOF 的催化下, 以苠基叠氮和苯 乙炔为原料, 在温和条件下高产率合成了 1,4-二取代三 氮唑衍生物, 催化反应机理研究表明, $\mathrm{Cu}$ 以二价态的形 式参与反应, 先形成炔基铜化合物, 再与苄基叠氮反应
生成三氮唑-5-铜盐, 最终又脱除 $\mathrm{Cu}^{2+}$ 得到产物并完成 了催化循环过程(Scheme 3).
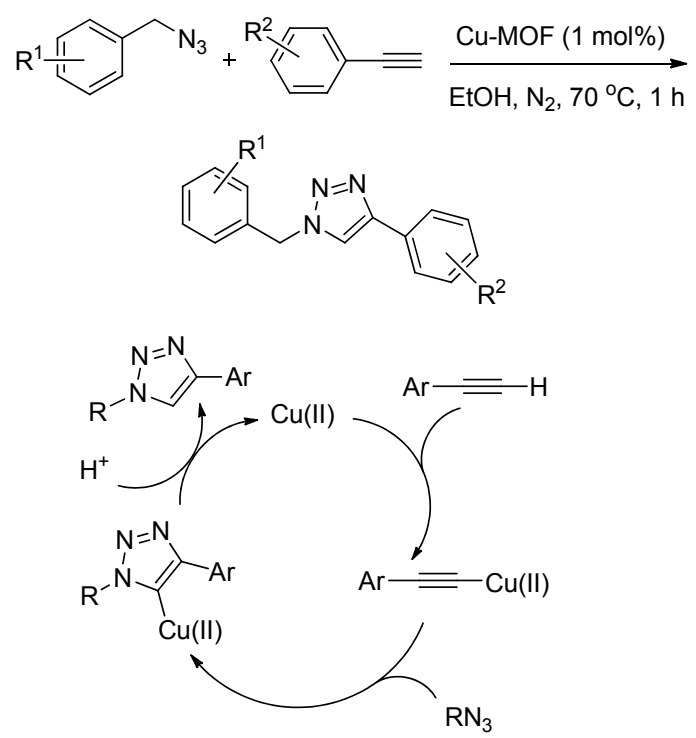

图式 $3 \mathrm{Cu}-\mathrm{MOF}$ 催化合成取代三氮唑的反应机理 Scheme 3 Mechanism of Cu-MOF catalyzed synthesis of substituted triazoles

碳氧键的构筑是合成含氧化合物的主要方法. 酚着 基的保护就是利用羟基与保护试剂间形成新的碳氧键 达到保护的目的. 2013 年, Phan 课题组 ${ }^{[34]}$ 用 2,2'-联吡啶6,6 '- 二羧酸 $\left(\mathrm{H}_{2} \mathrm{BPDC}\right)$ 和 $\mathrm{Cu}\left(\mathrm{NO}_{3}\right)_{2} \cdot 3 \mathrm{H}_{2} \mathrm{O}$ 合 成了 $\mathrm{Cu}_{2}(\mathrm{BPDC})_{2}(\mathrm{BPY})$ 型 $\mathrm{Cu}-\mathrm{MOF}$ ，并将其作为催化剂，以 邻着基苯甲醛和二氧六环为原料, 通过形成 $\mathrm{C}-\mathrm{O}$ 键实 现了酚羟基的保护(Eq. 10). 结果表明, $\mathrm{Cu}_{2}$ (BPDC) $)_{2}$ (BPY)作为催化剂能高效地催化该反应, 反应时间短, 仅需要 $1 \mathrm{~h}$, 且条件较温和, $100{ }^{\circ} \mathrm{C}$ 下就能完全反应, 产 率在 $95 \%$ 以上. 在该反应中二氧六环既是反应原料, 又 是溶剂. 值得一提的是, 该催化剂重复利用效果很好, 即使在重复使用 8 次后, 产率仍然在 $85 \%$ 以上.

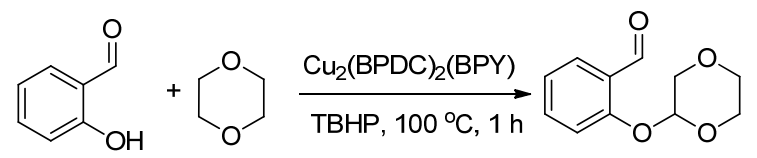

最近, Chen 课题组 ${ }^{[35]}$ 以 $\mathrm{Cu}(\mathrm{OAc})_{2} \cdot 2 \mathrm{H}_{2} \mathrm{O}$ 和 $2,4,6$-三 (4-吡啶基)-1,3,5-三嗪(TPT)为原料, 在 1-菜-2,6-二磺酸 二钠盐存在下合成了 $\mathrm{Cu}(\mathrm{I})-\mathrm{MOF}$. 研究发现, $\mathrm{Cu}(\mathrm{I})-\mathrm{MOF}$ 能高效地催化醛、环胺和炔烃的多组分反应(Eq. 11), 在 $80{ }^{\circ} \mathrm{C}$ 、无溶剂条件下生成了炔丙胺. 研究表明当催化剂 用量为 $1 \mathrm{~mol} \%$ 时，产率只有 $64 \%$; 逐步提高催化剂用量 可使产率升高，当达到 $2.5 \mathrm{~mol} \%$ 时产率达到 $91 \%$; 当继 续增加到 $10 \mathrm{~mol} \%$ 时，产率几乎无变化，为 $90 \%$. 该反 应条件简单温和，产率较高，可达到 $91 \%$ ，并且催化剂 
重复利用 5 次后产率仍在 $85 \%$ 以上. 另外, 芳香醛苯环 上不管是连有吸电子基团还是供电子基团, 对反应都几 乎无影响. 除了六元环胺外, 五元环的胺也能发生反应, 但产率相对于六元环要差一点, 而且受电子效应影响较 大, 芳香醛上连有供电子基团时产率能达到 $80 \%$, 连有 吸电子基团时产率降到 $62 \%$; 当与炔烃相连的芳香环连 有 $\mathrm{OCH}_{3}$ 时, 产率只有 $58 \%$.

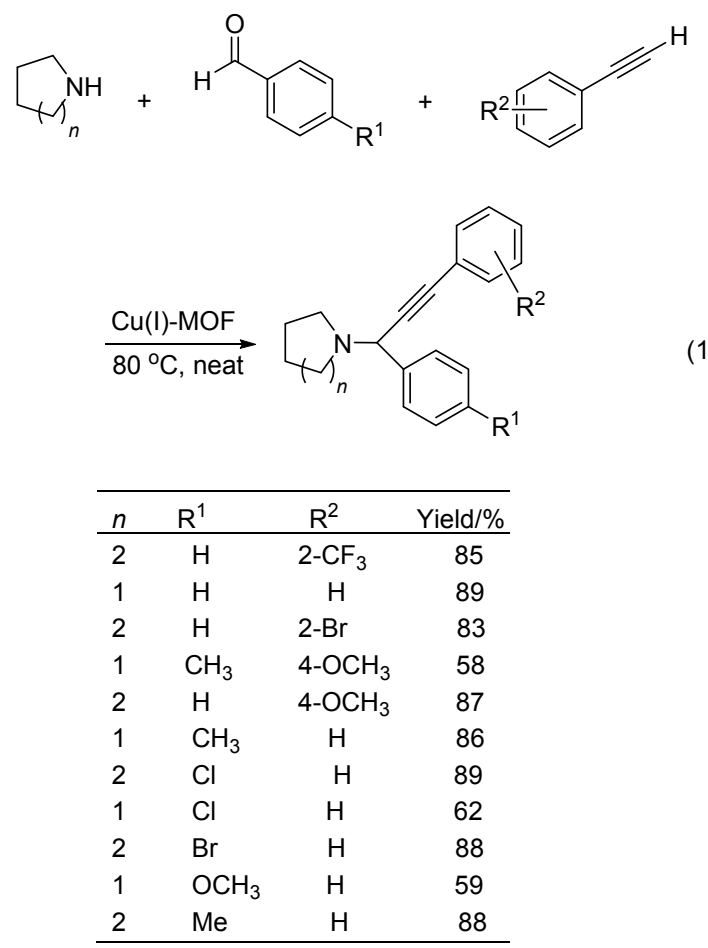

苯并噻唑是带有活性芳香环和具有大的共轭结构 的杂环芳香化合物, 在有机合成、医药合成和有机导电 聚合物方面具有重要的应用. Phan 等 ${ }^{[36]}$ 发现 $\mathrm{Cu}-\mathrm{MOF}$ 材 料在苯并噻唑类化合物的 $\mathrm{C}-\mathrm{H}$ 活化反应中具有很好的 催化效果. 2014 年, 该小组用 $\mathrm{Cu}\left(\mathrm{NO}_{3}\right)_{2} \cdot 3 \mathrm{H}_{2} \mathrm{O}$ 和 4,4'-联 吡啶 (BPY)为原料, 以 $4,4^{\prime}-$ 二苯醚二甲酸 $\left(\mathrm{H}_{2} \mathrm{OBA}\right)$ 为添 加剂制备了 $\mathrm{Cu}_{2}(\mathrm{OBA})_{2}(\mathrm{BPY})$ 型 Cu-MOF. 研究发现, $\mathrm{Cu}_{2}(\mathrm{OBA})_{2}(\mathrm{BPY})$ 能有效地催化苯并噻唑和碘苯发生芳 基化反应，高产率地生成 2-芳基苯并噻唑衍生物(Eq. 12). 在该反应中, 碘苯苯环上的电子效应对反应影响 较大, 碘苯上连有供电子基团对反应具有明显的促进作 用, 产率最高可达 $99 \%$; 而连接吸电子基团的阻碍作用 也很明显, 产率普遍下降, 部分产物的产率降至 53\%左 右.

端炔烃与其他化合物的偶联也是形成新的 $\mathrm{C}-\mathrm{C}$ 键 的有效方法. 通常情况下炔烃末端氢原子首先被金属取 代, 形成金属炔化物再进行亲核取代反应, 很少有报道 炔烃直接与其他化合物发生偶联反应. 2014 年 Phan

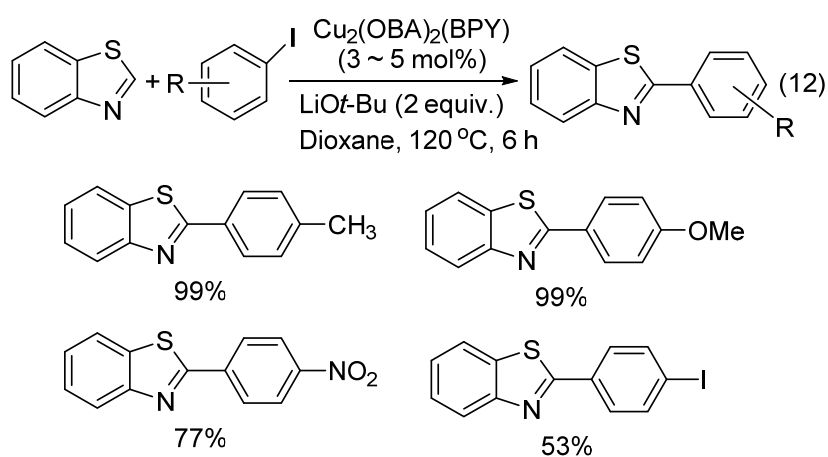

等 ${ }^{[37]}$ 以 $\mathrm{H}_{3} \mathrm{BTC}$ 和 $\mathrm{Cu}\left(\mathrm{NO}_{3}\right)_{2} \cdot 3 \mathrm{H}_{2} \mathrm{O}$ 为原料, 用相同的方 法制备了 $\mathrm{Cu}-\mathrm{MOF} 199$, 在它的催化下, $N, N$-二甲基苯胺 与芳基乙炔在一定条件下直接发生 $\mathrm{C}-\mathrm{C}$ 偶联反应( $\mathrm{Eq}$. $13)$, 产率最高能达到 $81 \%$. 这是一个氧化脱氢 $\mathrm{C}_{\mathrm{sp}^{3}}-\mathrm{C}_{\mathrm{sp}}$ 直接偶联的过程. 值得一提的是，除了芳基乙炔外，脂 肪族的炔烃也能发生该反应，不过反应产率没有芳基乙 炔高, 只有 $50 \%$ 左右. 另外, 若芳胺的苯环上连有吸电 子基团时由于苯环电子云密度降低反应产率只有 55\%.

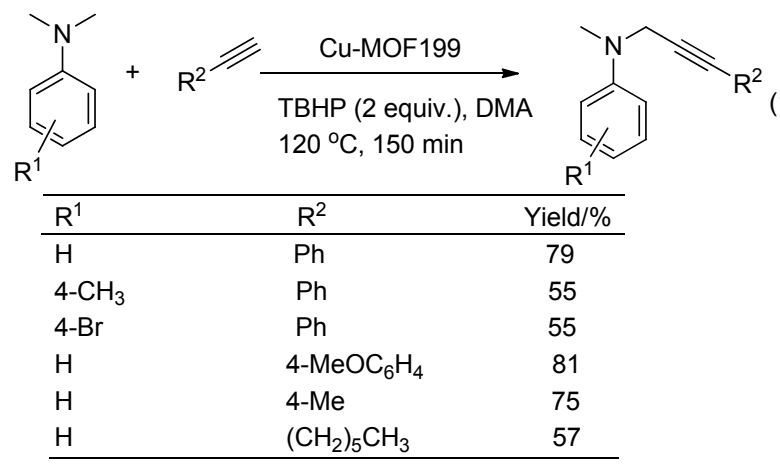

卤代烃与胺类化合物的反应是形成 $\mathrm{C}-\mathrm{N}$ 键的经典 反应. 2014 年 $\mathrm{Phan}$ 课题组 ${ }^{[38]}$ 用 $\mathrm{Cu}\left(\mathrm{NO}_{3}\right)_{2} \cdot 3 \mathrm{H}_{2} \mathrm{O}$ 和 $\mathrm{H}_{2} \mathrm{BDC}$ 作为原料, 以 1,4 二 二氮杂二环 [2.2.2] 辛烷 $(\mathrm{DABCO})$ 为添加剂制备了 $\mathrm{Cu}_{2}(\mathrm{BDC})_{2}(\mathrm{DABCO})$ 型 $\mathrm{MOF}$, 在该 $\mathrm{MOF}$ 的催化下卤代芳烃与咪唑发生 $N$-芳基化偶联 反应(Eq. 14), 反应时间大大缩短, 只需要 $3 \mathrm{~h}$ 就能完全 反应，部分产物的收率能达到 $100 \%$. 值得一提的是，当 在卤代烃上连有供电子基团时, 由于苯环电子云密度的 增大, 稳定了苯环与卤素的 P- $\pi$ 共轭体系, 致使反应产 率大大降低, 当连有 $\mathrm{NH}_{2}$ 时, 几乎不反应; 而咪唑环上 的取代基对该反应无明显影响.

最近 Phan 课题组 ${ }^{[39]}$ 用 Martínez 的方法 ${ }^{[28]}$ 制备了 Cu-MOF-74, 并在其催化下，2-羰基-2-苯基乙醛和胺在 敞开体系中就能发生交叉脱氢偶联反应, 生成相应的酰 胺 (Eq. 15). 当两物料比为 $1: 3$ 时, 反应产率能达到 $90 \%$ 以上. 五元和六元环仲胺能与 2-羰基-2-苯基乙醛发 生反应以很高的产率得到相应的产物; 当选用芳胺或链 
<smiles>[R]c1ccc(-n2ccnc2[R])cc1</smiles>

\begin{tabular}{llll}
\hline $\mathrm{X}$ & $\mathrm{R}$ & $\mathrm{R}^{1}$ & Yield/\% \\
\hline $\mathrm{I}$ & $4-\mathrm{OCH}_{3}$ & $\mathrm{H}$ & 58 \\
$\mathrm{I}$ & $4-\mathrm{NH}_{2}$ & $\mathrm{H}$ & Trace \\
$\mathrm{I}$ & $4-\mathrm{COCH}_{3}$ & $2-\mathrm{NO}_{2}$ & 100 \\
$\mathrm{I}$ & $4-\mathrm{COCH}_{3}$ & $2-\mathrm{CH}_{3}$ & 100 \\
$\mathrm{Br}$ & $4-\mathrm{NO}_{2}$ & $\mathrm{H}$ & 100 \\
$\mathrm{Br}$ & $3-\mathrm{NO}_{2}$ & $\mathrm{H}$ & 100 \\
$\mathrm{Br}$ & $2-\mathrm{NO}_{2}$ & $\mathrm{H}$ & 100 \\
$\mathrm{Br}$ & $4-\mathrm{OCH}_{3}$ & $\mathrm{H}$ & 53 \\
$\mathrm{Cl}$ & $\mathrm{H}$ & $\mathrm{H}$ & 100 \\
$\mathrm{Cl}$ & $4-\mathrm{NO}_{2}$ & $\mathrm{H}$ & 88 \\
$\mathrm{Cl}$ & $4-\mathrm{COCH}_{3}$ & $\mathrm{H}$ & 29 \\
\hline
\end{tabular}

状脂肪胺时反应产率只有 $50 \%$ 左右. 与传统方法相比, 该方法无需额外添加氧化剂和碱性化合物. 另外, 该催 化剂重复利用 9 次后产率仍然能达到 $80 \%$.<smiles>O=CC(=O)c1ccccc1</smiles>

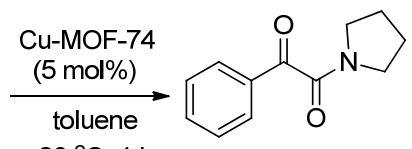<smiles>CC(C)(C)OC(=O)C(=O)N1CCCCC1</smiles><smiles>O=C(C(=O)N1CCOCC1)c1ccccc1</smiles><smiles>CC1CCN(C(=O)C(=O)c2ccccc2)CC1</smiles><smiles></smiles>

2015 年, Cho 课题组 ${ }^{[40]}$ 用 Cu-MOF199 作为催化剂, 在微波条件下催化 $\beta$-溴 $-\alpha, \beta$-不饱和羧酸和端炔的偶联
合环反应，目标产物的产率最高可达 85\% (Eq. 16). 研 究表明，该反应底物的适用范围很广，烯烃双键可以连 接在五元环、六元环、八元环、甚至十元环上，也可以 是多环烯烃, 如苯并环已烯, 烯烃环上还可以连接其他 取代基，如 $\mathrm{CH}_{3} 、 \mathrm{Ph}$ 等; 炔烃可以是芳基乙炔，也可以 是脂肪族炔烃，这对反应产率影响不大，产率能达到 $70 \%$ 以上; 但是当炔烃上带有异丁基时，由于空间位阻 的存在，使反应产率降为 54\%. 可见该方法对合成双环 烯醇式内酯具有独到之处.

\section{Zn-MOF 在催化有机反应中的应用}

具有共轭结构的化合物和骨架结构的 MOF 混合催 化也是多相催化反应研究的热点. 2009 年, Hupp 课题 组 ${ }^{[41]}$ 用 $\mathrm{Zn}\left(\mathrm{NO}_{3}\right)_{2} \cdot 6 \mathrm{H}_{2} \mathrm{O}$ 和 5,15-二吡啶-10,20-二五氟卟 啉为原料合成了以具有大共轭结构的卟啉为框架的 ZnPO-MOF 催化剂. 用该催化剂在乙腈中 $60{ }^{\circ} \mathrm{C}$ 下实现 了醇和 $N$-乙酰基咪唑的催化酯化反应得到乙酸酯, 同 时副产咪唑(Scheme 4).

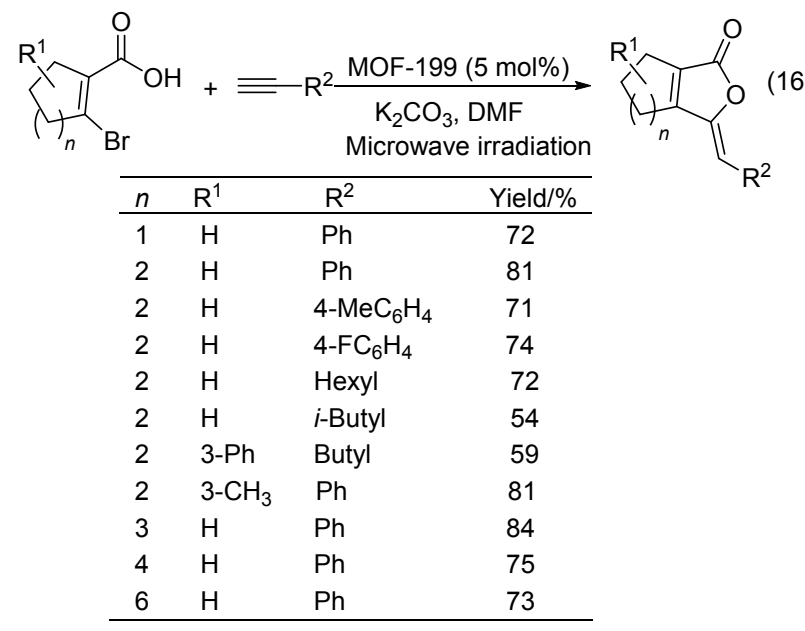

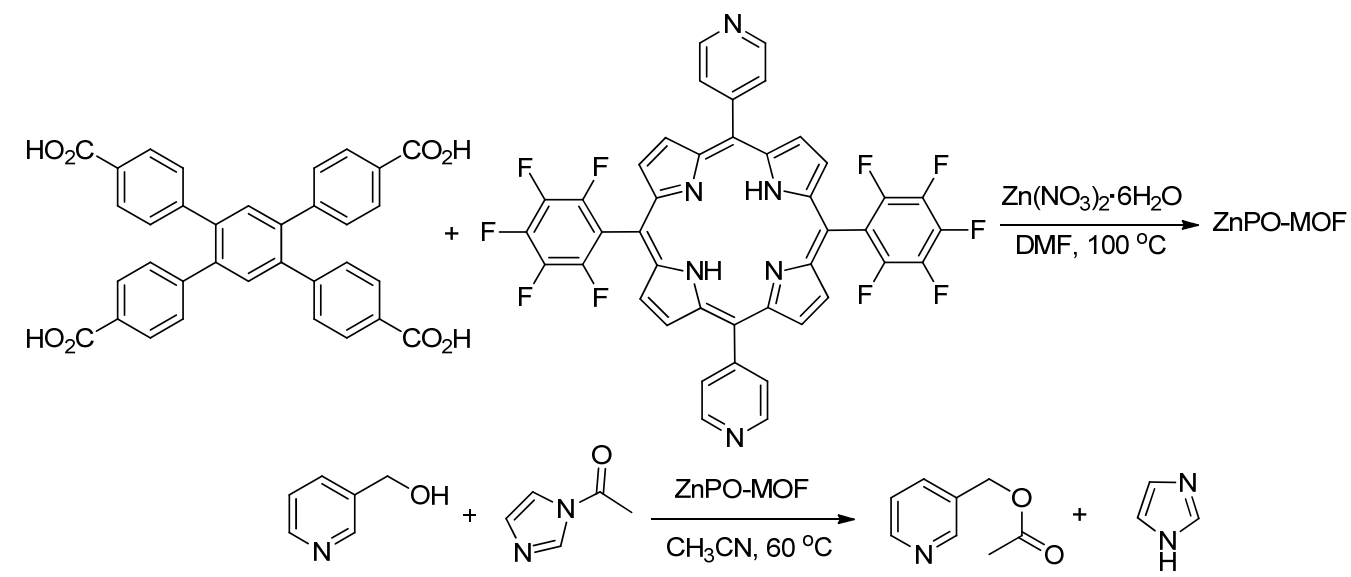

图式 $4 \mathrm{ZnPO}-\mathrm{MOF}$ 的制备及其在有机合成中的应用

Scheme 4 Preparation of ZnPO-MOF and its application in organic synthesis 
维蒂希(Witting)反应是羰基生成烯烃的经典反应, 反应条件简单，但反应原子经济性比较差 ${ }^{[42] .2009}$ 年, Bharadwaj 课题组 ${ }^{[43]}$ 以 $\mathrm{Zn}\left(\mathrm{NO}_{3}\right)_{2} \cdot 6 \mathrm{H}_{2} \mathrm{O}$ 为金属来源, 用 $\mathbf{L}$ 作为骨架制备了 Zn-MOF, 利用该 Zn-MOF 作为催化剂, 二氯甲烷为溶剂, 研究了醛与亚甲基化合物合成碳碳双 键的反应, 即 Knoevenagel 反应(Scheme 5), 该反应不 仅条件简单、温和, 而且原子经济性高, 是合成烯烃的 理想路线, 不足之处是, 亚甲基化合物仅限于㲵基取代 的烷烃. 另外, 芳醛与三甲基硅腈在该 Zn-MOF 催化下 也能发生反应得到氰基醇, 反应在 $0{ }^{\circ} \mathrm{C}$ 下进行, 只需 $1.5 \mathrm{~h}$ 即可完成; 当苯环上连有吸电子基团如 $\mathrm{NO}_{2}$ 时, 由 于强吸电子效应, 活化了苯环对位的醛基, 所以产率较 高, 在 $90 \%$ 以上, 如果是供电子基团如 $\mathrm{CH}_{3}$ 时, 产率会 降低到 $80 \%$ 左右.

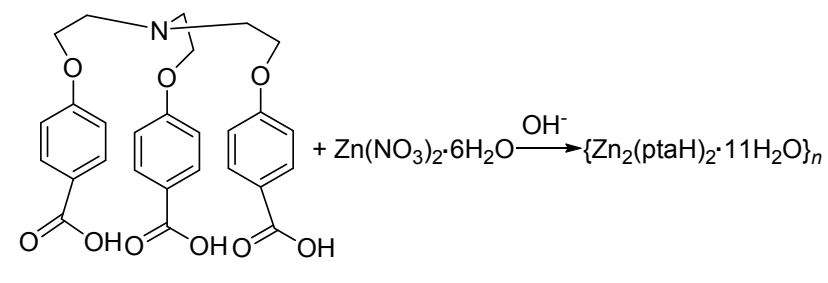

$\mathrm{L}$

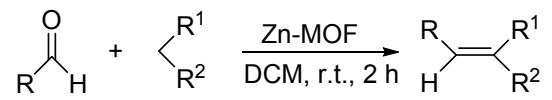<smiles>[R]c1ccc([CH+]C(=O)c2ccc([R])cc2)cc1</smiles>

图式 $5 \mathrm{Zn}-\mathrm{MOF}$ 参与的 Knoevenagel 反应

Scheme 5 Knoevenagel reaction by $\mathrm{Zn}-\mathrm{MOF}$

2010 年 Phan 课题组 ${ }^{[44]}$ 用 $\mathrm{Zn}\left(\mathrm{NO}_{3}\right)_{2} \cdot 6 \mathrm{H}_{2} \mathrm{O}$ 和均苯三 甲酸在 DMF 中, 用加热的方法制备了 $\mathrm{Zn}-\mathrm{MOF}$ 复合物. 利用该 Zn-MOF 作为 Lewis 酸催化剂, 通过傅克烷基化 反应以 $97 \%$ 的收率得到了甲苯对位取代的烷基化产物, 邻位烷基化产物仅占 $1 \%$ (Eq. 17). 该反应时间短, 效率 高, 区域选择性好.

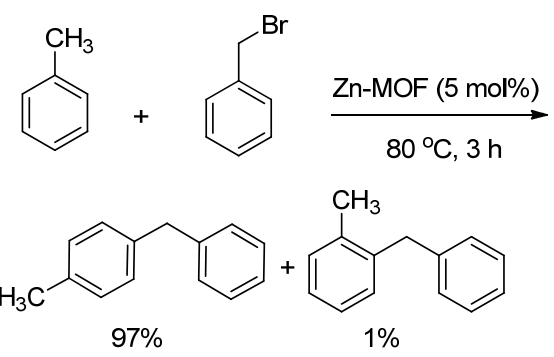

含有 $\alpha-\mathrm{H}$ 的酮一般在碱性条件下能与羰基化合物 发生羟醛缩合反应生成 $\beta$-差基酮, 但很少有报道在非碱
或非酸性条件下能直接生成 $\beta$-羟基酮. 2014 年 Duan 等 ${ }^{[45]}$ 用二甲基-5-(丙-2-炔氧基)间苯二甲酸，内消旋 $\alpha, \beta$ 二(4-吡啶基)乙二醇与 $\mathrm{ZnCl}_{2}$ 制备了手性 $\mathrm{Zn}-\mathrm{MOF}$ 复合 物. 研究发现, 在甲醇/水为混合溶剂的条件下, 该 $\mathrm{Zn}-\mathrm{MOF}$ 能催化芳醛和环己酮发生缩合反应，以很高的 立体选择性生成 $\beta$-差基酮(Eq. 18). 与传统的差醛缩合 反应相比，该反应不需要碱或酸性添加剂，而且反应溶 剂是甲醇/水，绿色环保、无毒无害，属于 $100 \%$ 原子经 济性的环境友好反应.

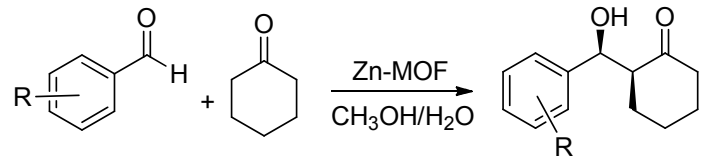<smiles>O=C1CCCCC1[C@H](O)c1ccc([N+](=O)[O-])cc1</smiles><smiles>O=C1CCCCC1[C@H](O)c1ccccc1[N+](=O)[O-]</smiles>

$e e=59 \%$

\section{$3 \mathrm{Ni}-$ MOF 在有机合成中的应用}

2012 年, Phan 等 ${ }^{[46]}$ 用 $\mathrm{Ni}\left(\mathrm{NO}_{3}\right)_{2} \cdot 6 \mathrm{H}_{2} \mathrm{O} 、 4,4^{\prime}$-联二吡 啶和 $\mathrm{H}_{3} \mathrm{BTC}$, 以共加热法制备了 $\mathrm{Ni}(\mathrm{HBTC})(\mathrm{BPY})$ 型 Ni-MOF. 研究发现该 MOF 对苯硼酸和芳香醛的亲核加 成反应具有很好的催化效果. 研究发现, 在甲苯溶液中, 在 $100{ }^{\circ} \mathrm{C}$ 下反应 $6 \mathrm{~h}$, 就能得到醛的亲核加成产物, 而 且产率高达 $95 \%$. 该方法对于其它取代的苯嗍酸和取代 的芳香醛也有很好的适用性(Eq. 19). 当芳香醛连有供 电基团时有助于反应的进行，当连有 $\mathrm{OCH}_{3}$ 时，由于受 到 $\mathrm{p}-\pi$ 共轭效应和供电子效应的共同影响，导致产率只 有 $43 \%$ ，当连有吸电子基团时对反应有一定的阻碍作 用; 当苯硼酸上有较弱的吸电子基团时对反应有利，同 时供电子基团有助于该反应的进行. 另外, 该催化剂重 复利用 5 次后，产率保持在 $80 \%$ 左右.

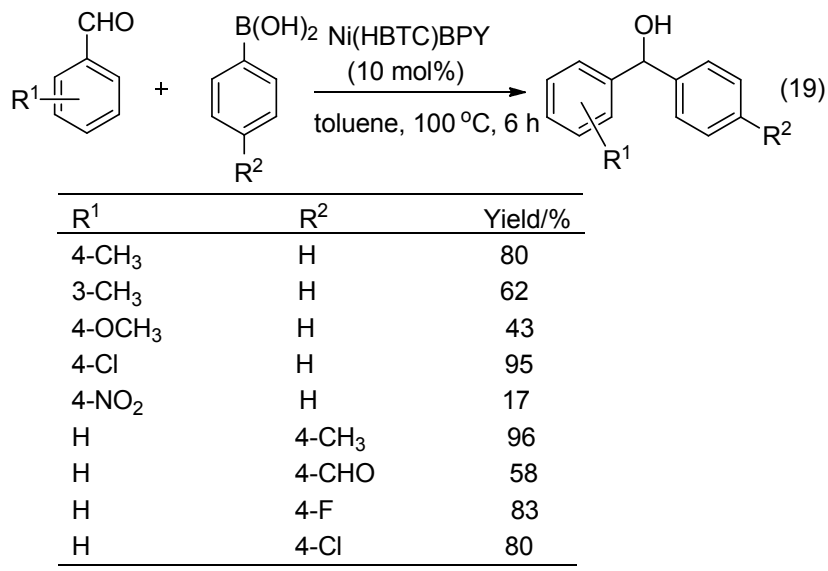


2014 年, 王训等 ${ }^{[47]}$ 以对苯二甲酸 $\mathrm{H}_{2} \mathrm{BDC}$ 为有机连 接框架, 以 $\mathrm{Ni}^{2+}$ 和 $\mathrm{Zn}^{2+}$ 同时作为金属结点, 用二甲基乙 酰胺 (DMAC) 和乙醇作为混合溶剂制备了混和金属 $\mathrm{Zn}(\mathrm{Ni})$-MOF5. 作者研究了该 MOF 对碘代芳烃的羰化 酯化反应的催化性能, 结果发现碘苯、一氧化碳和乙醇 钠在乙醇中 $60{ }^{\circ} \mathrm{C}$ 下反应 $24 \mathrm{~h}$ 就能得到羰化酯化产物 (Scheme 6), 产率高达 $95 \%$, 当碘苯上连有吸电子基团 时反应产率有所下降. 相对于传统的醇酸酯化反应, 该 方法简单, 条件温和, 原料廉价易得, 产物收率高, 并 且避免了使用高危、高腐蚀性浓硫酸, 不会对环境造成 污染.

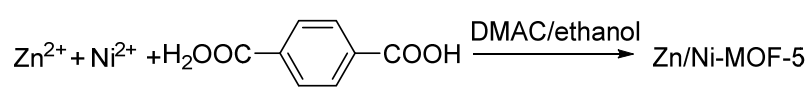

$$
\underbrace{\mathrm{Zn/Ni-MOF-5}}_{\mathrm{R}=\mathrm{H}, \mathrm{CH}_{3}, \mathrm{OCH}_{3}, \mathrm{~F}, \mathrm{NO}_{2}}
$$

图式 $6 \mathrm{Zn} / \mathrm{Ni}-\mathrm{MOF}-5$ 的制备及应用

Scheme 6 Preparation and application of $\mathrm{Zn} / \mathrm{Ni}-\mathrm{MOF}-5$

\section{Pd-MOF 在有机合成中的应用}

钯作为贵金属催化剂, 一般是在配体的配合下使用 的. 若单独使用钯化合物作催化剂而不加配体, 除个别 报道外 ${ }^{[48]}$, 往往不会表现出高效的催化性能. 直接以二 价钯作为金属节点用于含钯 MOF 复合物制备并用于催 化反应的文献鲜有报道. 但钯负载在其它金属形成的 MOF 上往往能表现出优异的催化性能.

Yaghi 课题组 ${ }^{[49]}$ 于 1999 年制备了一种具有特殊结构 的 $\mathrm{MOF}$, 是以 $\mathrm{Zn}_{4} \mathrm{O}_{6}^{+}$为金属中心, 以对苯二甲酸苯基酯 为骨架的立方网络结构, 称为 MOF-5. 2008 年 Klemm 课 题组 ${ }^{[50]}$ 用 MOF- 5 和硝酸钯在三乙胺和 DMF 的混合溶剂 中用共沉淀法制备了以 MOF-5 为载体, Pd(II)负载的 Pd/MOF-5 多相催化剂. 将该催化剂用于肉桂酸乙酯的 催化氢化反应中, 结果发现 Pd/MOF-5 能高效、高选择 性的催化肉桂酸乙酯中双键的还原, 产率达 $100 \%$, 但 不会还原苯环和酯基(Eq. 20). 值得一提的是, 该反应中 无需加入配体, 只需在有氢源的情况下就能高产率的得 到相应产物. 另外, 与传统的 $\mathrm{Pd} / \mathrm{C}$ 催化的加氢反应相 比, 该负载催化剂的催化活性更高, 而且能反复使用 5 次以上.

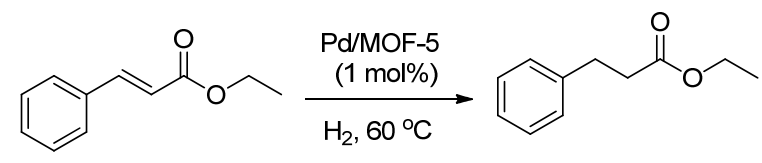

2010 年, 舒谋海研究组 ${ }^{[1]}$ 首先以 $\mathrm{Zn}\left(\mathrm{NO}_{3}\right)_{2} \cdot 6 \mathrm{H}_{2} \mathrm{O}$ 和
$\mathrm{H}_{2} \mathrm{BDC}$ 为原料制备了 MOF-5 ${ }^{[52]}$, 同时将 $\mathrm{PdCl}_{2}$ 和 $\mathrm{NaCl}$ 溶于 DMF 中, 再向其中加入 MOF- 5 , 经水合肼还原后 得到了负载型 $\mathrm{Pd} / \mathrm{MOF}-5$. 新制的 $\mathrm{Pd} / \mathrm{MOF}-5$ 在空气中易 自燃, 因此需在 $\mathrm{N}_{2}$ 保护下使用. 研究发现, 在该 Pd/MOF-5 的催化下, 碘苯与苯乙炔可以很容易的发生 Sonogashira 偶联反应，各种取代的碘苯、取代的芳香炔 烃、脂肪族炔烃参与反应都能获得非常好的结果, 产率 在 95\% 99\%之间(Eq. 21). 该催化剂在重复使用 2 次时 产率仍在 $92 \%$ 以上; 但当用到第三次时, 产率会显著下 降到 $60 \%$.
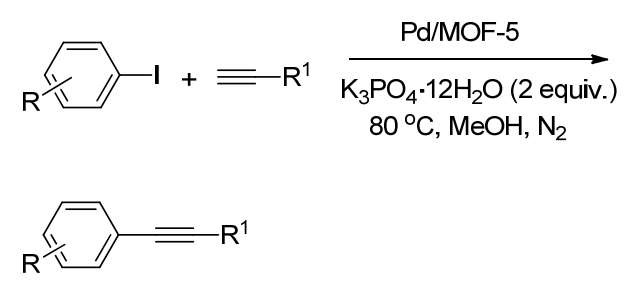

卤代烃在有机合成和工业生产中有重要应用, 而对 卤代烃的还原脱卤反应既是化学转化的需要, 更具有环 境中有机卤化污染物降解消除的现实意义. 2014 年 Ma 等 ${ }^{[53]}$ 利用 $\mathrm{Fe}\left(\mathrm{NO}_{3}\right)_{3} \cdot 9 \mathrm{H}_{2} \mathrm{O}$ 和反丁烯二酸制备了 $\mathrm{Fe}-\mathrm{MOF}$, 将 $\mathrm{PdCl}_{2}$ 溶于 $\mathrm{KCl}$ 溶液中形成 $\left[\mathrm{PdCl}_{4}\right]^{2-}$ 复合物, 再加入 $\mathrm{Fe}-\mathrm{MOF}$ 制得了负载型的 $\mathrm{Pd} / \mathrm{Fe}-\mathrm{MOF}$, 并将其应用到卤 代烃的还原反应中, 取得了很好的效果(Eq. 22). 反应以 该 $\mathrm{Pd} / \mathrm{Fe}-\mathrm{MOF}$ 化合物为催化剂, 在氢氧化钠水溶液中 通入 $101 \mathrm{kPa}$ 的氢气，即可脱去卤代烃中的卤原子而得 到芳香烃化合物，产率高达 99\%. 同样条件下，多卤代 烃也能被逐一完全还原, 但却不会将苯环还原. 显然这 是一种高效的选择性脱卤方法.
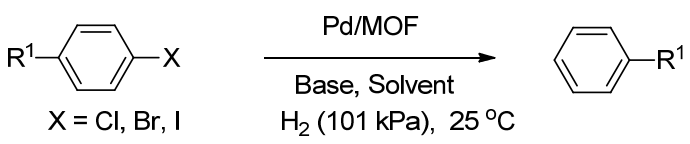

(22)

Ullmann 偶联反应和 Suzuki-Miyaura 偶联反应都是 生成联苯的经典反应，但很少报道一种催化剂能同时催 化这两类反应. 2015 年, $\mathrm{Li}$ 课题组 ${ }^{[54]}$ 用 $\mathrm{AlCl}_{3} \cdot 6 \mathrm{H}_{2} \mathrm{O}$ 和 $2,2^{\prime}$-联吡啶 $-5,5^{\prime}$-二羧酸为原料制备了 Al-MOF $253^{[55]}$, 用该 $\mathrm{MOF}$ 和 $\mathrm{PdCl}_{2}$ 为原料, 在乙腈和 $\mathrm{DMF}$ 的混合溶剂 中反应, 制备了负载型的催化剂 $\mathrm{Pd} / \mathrm{Al}-\mathrm{MOF} 253$. 研究 发现, Pd/Al-MOF253 既是 Ullmann 偶联反应也是 Suzuki-Miyaura 偶联反应的高效催化剂, 而且不管在卤 代芳烃和芳基硼酸上连有吸电子基团还是供电子基团, 对反应都几乎无影响, 反应产率都能达到 $80 \%$ 以上 (Scheme 7).

近期, Liu 课题组 ${ }^{[5]}$ 用制备的 $\mathrm{Cu}(\mathrm{DBC})$ 作为载体, 在亚胺型 $\mathrm{PySI}$ 配体和 $\mathrm{PdCl}_{2}$ 存在下, 制备了负载型的钯 

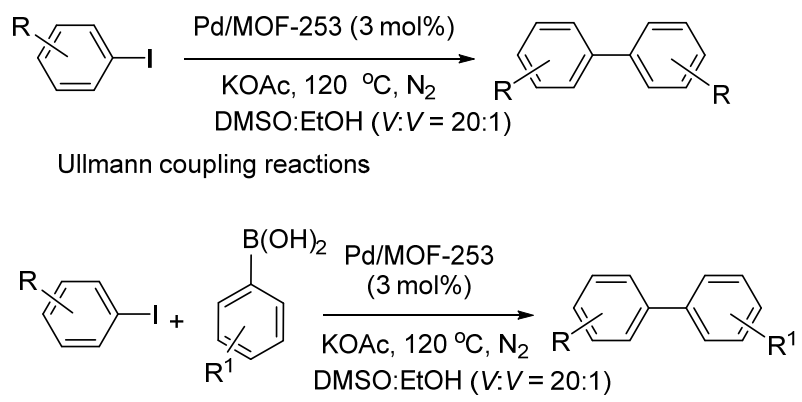

Suzuki-Miyaura couplings reactions

图式 $7 \mathrm{Pd} / \mathrm{Al}-\mathrm{MOF} 253$ 催化的偶联反应

Scheme 7 Pd/Al-MOF253 catalyzed coupling reaction 纳米催化剂 Pd@Cu-BDC/PySI, 其平均粒径为 $260 \mathrm{~nm}$. 研究发现, 该催化剂能高效的催化 Suzuki 反应, 反应条 件温和简单，反应时间短，产物收率高，取代基的电子 效应对该反应几乎无影响(Scheme 8).

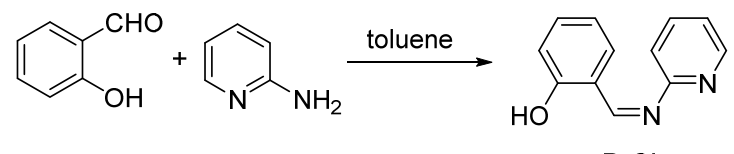

PySI

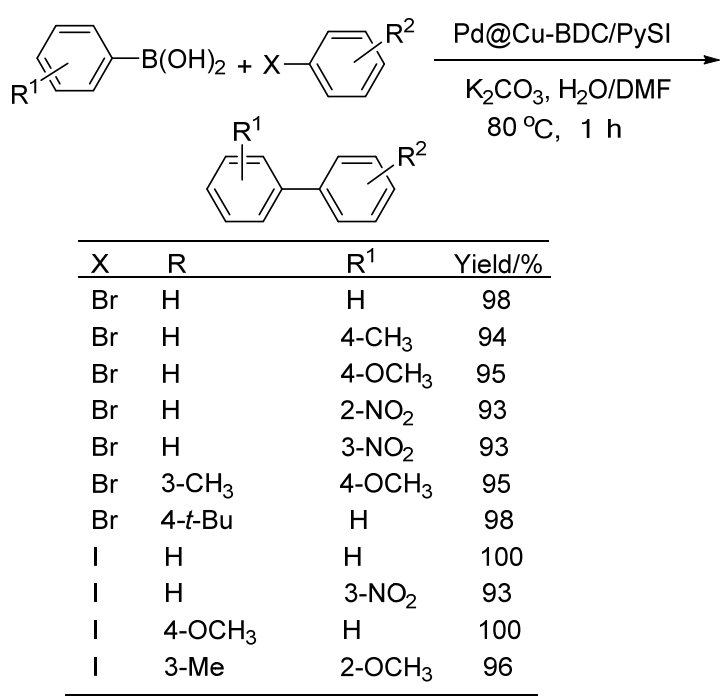

图式 8 PySI 的制备及 Pd@Cu-BDC/PySI 催化剂的应用 Scheme 8 Preparation of PySI and the application of $\mathrm{Pd} @ \mathrm{Cu}-$ BDC/PySI catalyst

除了负载型的钯 MOF 外, 也有以钯为金属节点的 Pd-MOF 的报道. 2014 年 Lin 课题组 ${ }^{[57]}$ 用 2,2'-联吡啶和 $\left[\mathrm{Pd}\left(\mathrm{CH}_{3} \mathrm{CN}\right)_{4}\right]\left(\mathrm{BF}_{4}\right)_{2}$ 为原料, 制备了 $\mathrm{Pd}-\mathrm{MOF}$, 发现氧气 条件下能使环已烯酮脱氢氧化成苯酚(Scheme 9), 产率 为 $81 \%$, 而且当环己烯酮连有给电子基团时能大大提高 反应效率, 可以定量地得到产物. 研究表明溶剂对产率 的影响很大, 例如, 当 $\mathrm{R}$ 为 $\mathrm{CH}_{3}$ 时, 在甲苯中产率为 $80 \%$, 在二甲苯中只有 $39 \%$, 但在 DMSO 中却可以达到
$100 \%$.<smiles>[R]C1=CC(=O)CCC1</smiles><smiles>CCc1cccc(O)c1</smiles>

图式 9 Pd-MOF 的制备及应用

Scheme 9 Preparation and application of Pd-MOF

\section{Zr-MOF 在有机合成中的应用}

2013 年 Marta 课题组 ${ }^{[58]}$ 用 $\mathrm{ZrCl}_{4}$ 和 $\mathrm{H}_{2} \mathrm{BDC}$ 在一定 条件下制得了 Zr-MOF(原文献编号为 UiO-66-LIr)，并利 用其作为催化剂，研究异丙醇中醛与硝基苯的交叉偶联 反应. 结果表明该反应可得到相应的 $\mathrm{N}$-烷基化产物，产 率在 $95 \%$ 以上. 反应过程被认为是，硝基苯先被还原成 胺, 胺与醛生成亚胺, 亚胺再被还原成氮的烷基化产物 (Scheme 10). 反应中醛成了烷基化试剂，异丙醇既是溶 剂又是氢供体. 该反应以工业来源丰富的硝基化合物为 原料，价格低廉，产物收率高，是一种有效的 $\mathrm{N}$-烷基化 反应的方法.
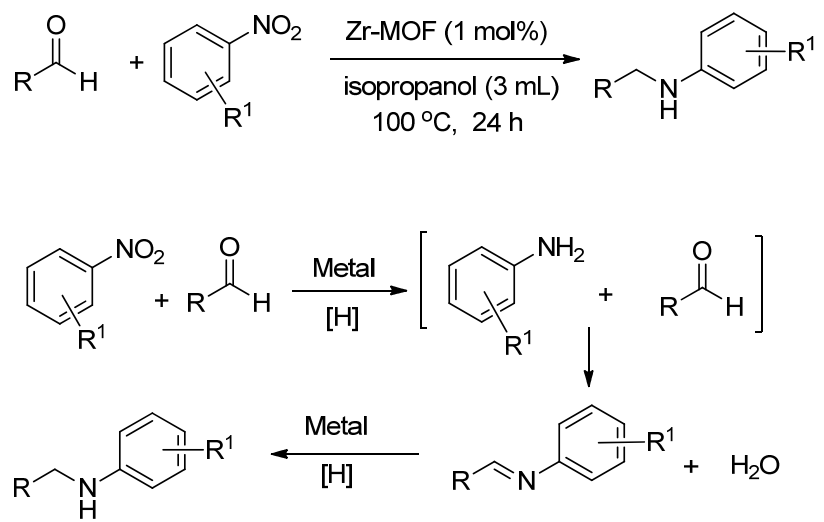

图式 $10 \mathrm{Zr}-\mathrm{MOF}$ 催化的硝基化合物与醛的 $N$-烷基化反应及 其机理

Scheme $10 N$-Alkylation of nitro compounds with aldehydes catalyzed by Zr-MOF and the mechanism

2014 年 Ren 课题组 ${ }^{[59]}$ 用与 Marta 课题组 ${ }^{[58]}$ 相同的 方法制备了 Zr-MOF，通过探索发现在 Zr-MOF 的催化 
下酰胺能发生 $\mathrm{C}-\mathrm{N}$ 键的断裂, 生成胺和羧酸. 该反应 在室温下进行 $5 \mathrm{~h}$ 就能高效的完成, 除了催化剂, 不需 要添加其他任何助剂, 产率高达 99\%. 更重要的是一向 被认为很稳定的 $N, N$-二取代甲酰胺在该条件下一样可 被分解为甲酸和相应的胺(Eq. 23).

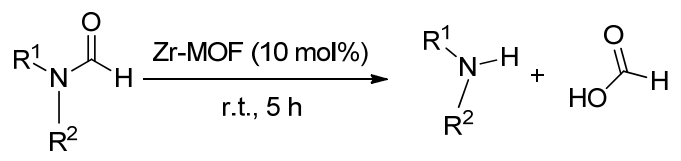

\section{$6 \mathrm{Cr}-\mathrm{MOF}$ 在有机合成中的应用}

2014 年 Janiak 课题组 ${ }^{[60]}$ 用取代的 $\mathrm{H}_{2} \mathrm{BDC}$ 、四甲基 氢氧化铵 $(\mathrm{TMAOH})$ 和 $\mathrm{Cr}\left(\mathrm{NO}_{3}\right)_{3} \cdot 9 \mathrm{H}_{2} \mathrm{O}$ 在高压反应釜中 反应, 制得了平均粒径为 $20 \mathrm{~nm}$ 的 Cr-MOF 催化剂, 大 大增加了催化剂的比表面积, 使其具有更高的催化活 性. 研究发现, 该 Cr-MOF 在 $70{ }^{\circ} \mathrm{C}$ 下能高效地催化缩 醛化反应, $1.5 \mathrm{~h}$ 就能使原料反应完全, 具有条件温和、 反应时间短、产物收率高的特点(Eq. 24). 电子效应对该 反应影响不大, 醛的芳环上不管是供电子基团还是吸电 子基团, 都能得到较高的产率. MOF 催化剂的配体上连 接的基团不同, 配体的酸性不同, 对反应的催化效果也 有不同, 配体的酸性大小顺序为 $\mathrm{R}=\mathrm{NO}_{2}>\mathrm{R}=\mathrm{H}>\mathrm{R}=$ $\mathrm{NH}_{2}$.

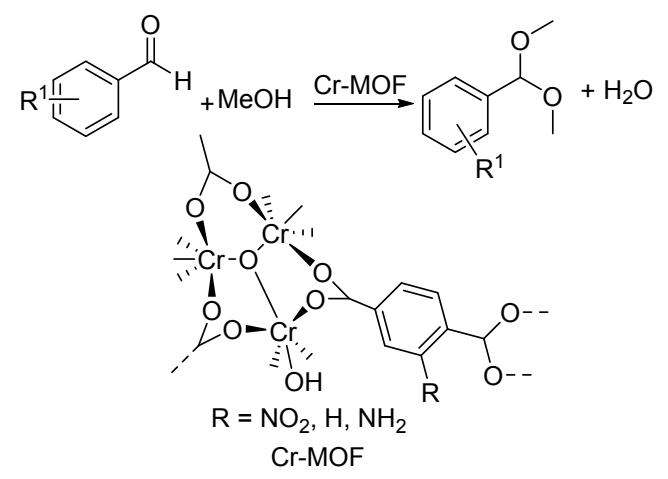<smiles>COC(OC)c1ccccc1</smiles>

$96 \%$<smiles>COC(OC)c1ccc(C)cc1</smiles>

$97 \%$<smiles>COC(OC)c1ccc(Cl)cc1</smiles>

$98 \%$<smiles>COC1(OC)CCCCC1</smiles>

$84 \%$<smiles>COC(C)(OC)C1CCCCC1</smiles>

$4 \%$
我们知道配体在过渡金属催化反应中具有很重要 的作用. 但以手性配体与金属自组装形成的 MOFs 材料 用作多相反应的催化剂却鲜有报道. 2014 年 Gascon
等 ${ }^{[61]}$ 用 $L$-脯氨酰胺为手性配体(L1), 金属铬为金属结 点, 首先制备了手性 Cr-MOF101, 经过酸化, 得到了具 有手性的金属骨架 (S)-Cr-MOF101。研究者发现, (S)-Cr-MOF101 能高效的催化羟醛缩合反应(Eq. 25), 得 到 $(S)$-构型的手性产物，对映体过量值不低于 $90 \%$. 取 代基的电子效应对该反应影响不大, 不管是吸电子基团 还是供电子基团, 产率都在 $90 \%$ 以上.

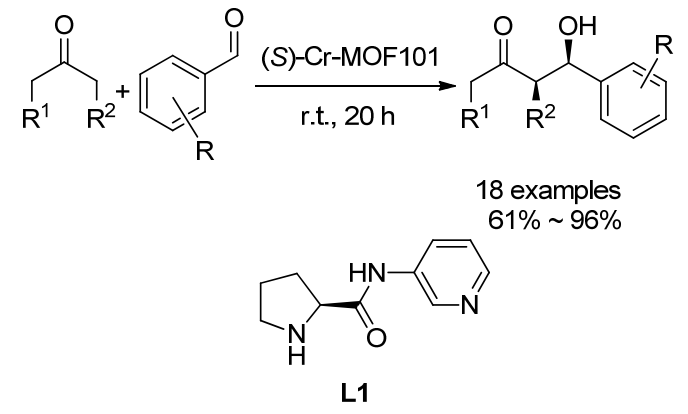

醛类化合物是重要的有机合成中间体，其高效的合 成方法自然备受关注. Saikia 课题组 ${ }^{[62,63]}$ 利用 $\mathrm{H}_{2} \mathrm{BDC}$ 和 $\mathrm{Cr}\left(\mathrm{NO}_{3}\right)_{3} \cdot 9 \mathrm{H}_{2} \mathrm{O}$ 反应制备了 $\mathrm{Cr}-\mathrm{MOF} 101$, 并将其负载到 $\mathrm{Fe}_{3} \mathrm{O}_{4}$ 上, 得到具有磁性的 Cr-MOF101@ $\mathrm{Fe}_{3} \mathrm{O}_{4}$. 该材料 能均匀的分散在乙醇当中, 当受到磁铁的吸引时立即缩 成一团利于回收. 以此作为催化剂, 以叔丁基过氧化氢 为氧化剂, 伯醇能很容易的被氧化成醛. 该反应条件温 和、产率高、时间短，催化剂可重复使用 6 次而产物的 收率没有明显下降, 并且不受电子效应的影响(Eq. 26).

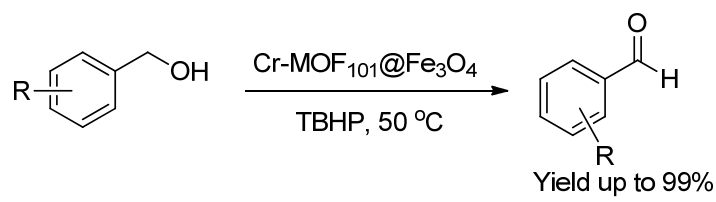

\section{Fe-MOF 在有机合成中的应用}

烯烃的还原一般是在贵金属催化下进行，很少报道 用廉价金属催化的烯烃还原反应. 2014 年 Lin 等 ${ }^{[64]}$ 经过 三个过程, 合成了含有水杨酫亚胺 $(\mathrm{sal})$ 的配体(L2), 并 以此为原料和廉价的 $\mathrm{FeCl}_{2} \bullet 4 \mathrm{H}_{2} \mathrm{O}$ 制备了 sal-Fe-MOF${ }^{[55]}$. 在该复合材料中, 金属原子与亚胺 $\mathrm{N}$ 原子和羟基 $\mathrm{O}$ 原子 相连形成稳定络合物而参与反应. 以 sal-Fe-MOF 为催 化剂, 在通入氢气的条件下, 烯烃在四氢呋喃中室温下 反应 $18 \mathrm{~h}$ 就能被顺利还原, 链烯和环烯也不例外, 产率 高达 $95 \%$ (Scheme 11). 不过该方法也有缺点, 即氢气的 压力太大，需要在 $4.04 \mathrm{MPa}$ 下操作.

2014 年 Kikhtyanin 课题组 ${ }^{[65]}$ 用 $\mathrm{H}_{2} \mathrm{BDC}$ 和硝酸铁制 备了 Fe-MOF, 在 Fe-MOF 的催化下, 糠醛和甲基酩能发 生羟醛缩合反应生成 $\alpha, \beta$-不饱和酮(Eq. 27). 与传统羟醛 

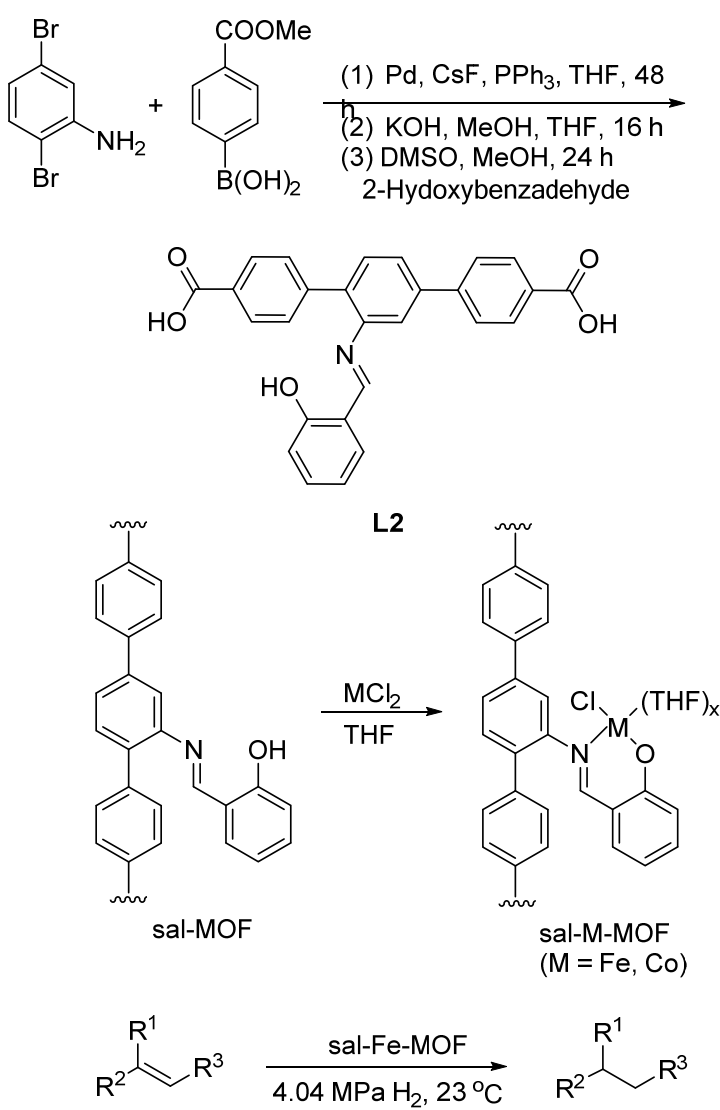

图式 11 sal-Fe-MOF 的制备及其参与的烯烃氢化反应

Scheme 11 Preparation of sal-Fe-MOF and the hydrogenation of alkenes

缩合反应相比, 该方法不需要添加碱性试剂, 在相对温 和的条件下就能以 $95 \%$ 的产率得到产物, 是合成带有活 性基团的羰基化合物的重要而有效的方法.

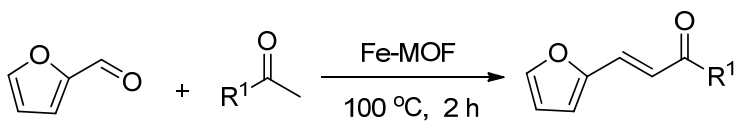

最近 Lin 课题组 ${ }^{[66]}$ 用取代的四联苯为原料, 经过三 步反应制备了具有手性的 Fe-MOF 催化剂, 研究发现该 $\mathrm{Fe}-\mathrm{MOF}$ 能催化分子内 $\mathrm{C}-\mathrm{H}$ 键的胺化反应得到具有手 性的环胺化合物(Scheme 12). 当 $\mathrm{X}$ 为 $\mathrm{CH}_{2}$ 时取代基的空 间位阻对该反应影响较大. 当 $\mathrm{X}$ 为 $\mathrm{O}$ 时, 铁的负载量为 $5 \%$ 时，产率为 $59 \%$ (a); 负载量为 $2 \%$ 时，产率为 $50 \%$ (b), 由此可见，铁的负载量对反应有一定的影响.

\section{8 其他 MOF 在有机合成中的应用}

$\mathrm{C}-\mathrm{H}$ 活化是近年来有机化学研究的热点之一, 是 直接构建碳碳键、碳杂原子键的原子经济的有效方法. 2014 年 Lin 课题组 ${ }^{[57]}$ 用 $2,2^{\prime}$-联吡啶和 $[\operatorname{Ir}(\mathrm{COD})(\mathrm{OMe})]_{2}$
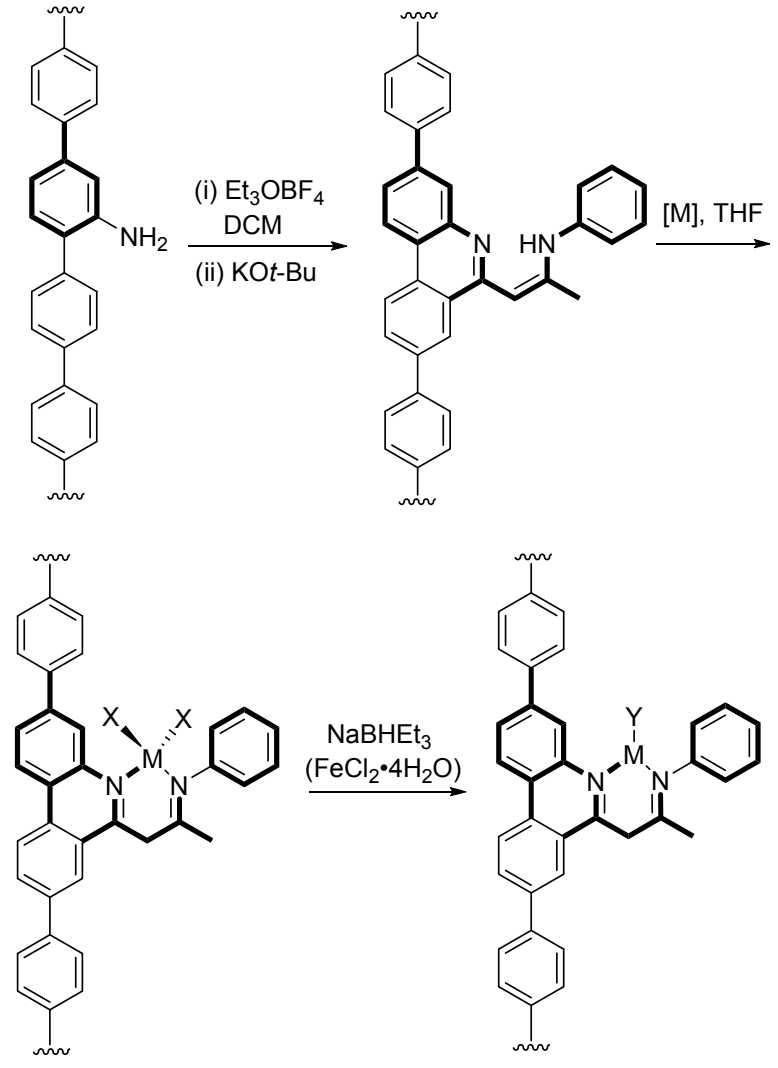

$\left.Y_{R^{2}}^{R} \sim_{N_{3}} \frac{\text { Fe-MOF }}{\mathrm{Boc}_{2} \mathrm{O}, \mathrm{C}_{6} \mathrm{H}_{6}} \underset{\mathrm{R}^{2}}{\longrightarrow} \mathrm{X}_{\mathrm{X}}^{\mathrm{N}}\right]$

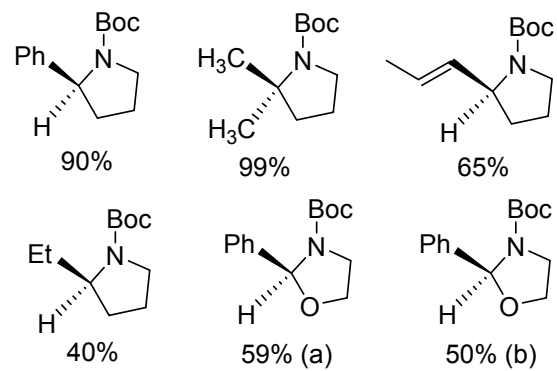

图式 12 Fe-MOF 的制备及应用

Scheme 12 Preparation and application of Fe-MOF

为原料制备了 Ir-MOF. 研究发现, 在庚烷为溶剂的条件 下 Ir-MOF 能高效的催化芳烃与联嗍酸频那醇酯发生 $\mathrm{C}-\mathrm{H}$ 活化反应生成两分子的芳基硼酸酯化合物，反应 一般发生在对位，产率高达 96\%，取代基的电子效应对 该反应无影响. 此外该催化剂还能催化 $\alpha$-取代的苯甲基 硅醚的芳烃 $\mathrm{C}-\mathrm{H}$ 活化硅烷基化合环反应，产率高达 $94 \%$; 但当 $\alpha$-位为苯基时，由于存在较大的空间位阻, 反应产率降为 $83 \%$ (Scheme 13).

2015 年 Bouchard 等 ${ }^{[67]}$ 以 $\mathrm{ZrOCl}_{2} \cdot 8 \mathrm{H}_{2} \mathrm{O}$ (氧氯化锆) 和内消旋-四(4-羧基苯基)卟吩氯化锰(TCPP-Mn)为原料 


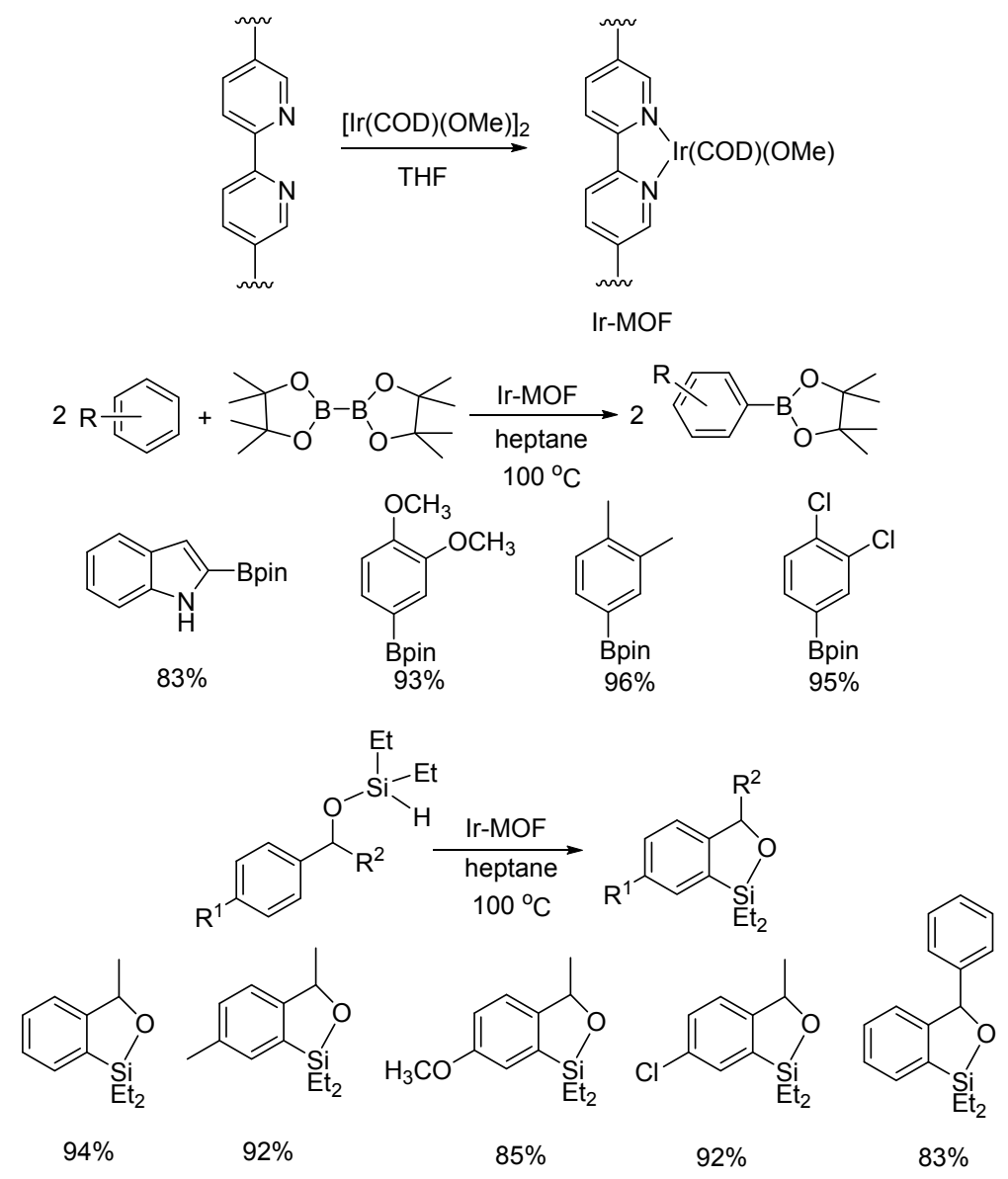

图式 13 Ir-MOF 的制备及其应用

Scheme 13 Preparation and application of Ir-MOF

制备了 Mn-MOF525. 研究发现, 该复合材料能催化烯 烃的环氧化反应, 产率高达 97\% (Eq. 28). 该反应在室 温下进行, 以氧气为氧化剂, 在 $2 \mathrm{~h}$ 的较短时间内即可 完成. 烯烃的适用范围很广, 双键可以在链段末端也可 以在中间, 也可以是环状烯烃. 相比较而言, 该催化体 系对链烯烃的催化环氧化的效果要比环烯烃差, 产率大 约只有 75\%. 另外, Mn-MOF525 重复利用 6 次后其催化 能力依然保持不变. 这与 Baiker 等 ${ }^{[24]}$ 制备的 $\mathrm{Cu}-\mathrm{MOF}$ 催 化烯烃的环氧化反应相比, 条件更温和, 底物适用范围 更广泛，可以扩展到链状烯烃.

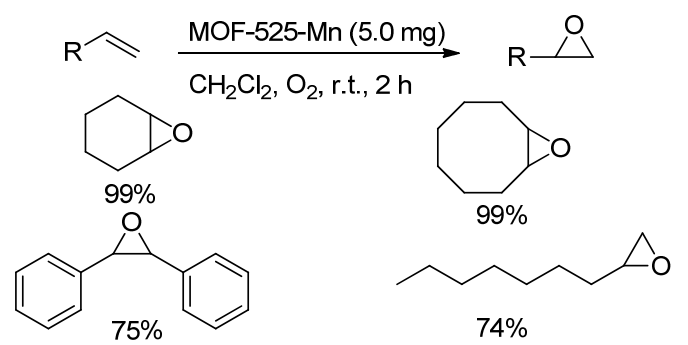

2015 年, Ren 课题组 ${ }^{[68]}$ 用 $\mathrm{H}_{3} \mathrm{BTC}$ 和 $\mathrm{Er}\left(\mathrm{NO}_{3}\right)_{3} \cdot 6 \mathrm{H}_{2} \mathrm{O}$ 为原料, 乙二胺为添加剂, 制备了 $\operatorname{Er}(\mathrm{btc})(\mathrm{ED})_{0.75^{-}}$
$\left(\mathrm{H}_{2} \mathrm{O}\right)_{0.25}$, 研究发现该催化剂也能催化 Knoevenagel 反 应生成烯烃(Eq. 29), 产率高达 99\%. 与 Bharadwaj 课题 组 ${ }^{[43]}$ 不同的是, Ren 课题组以环己烷为溶剂, 在 $40{ }^{\circ} \mathrm{C}$ 下 才能反应, 但是, $\mathrm{Er}(\mathrm{btc})(\mathrm{ED})_{0.75}\left(\mathrm{H}_{2} \mathrm{O}\right)_{0.25}$ 能重复利用 3 次, 产率能达到 $96 \%$.

$$
\left.\mathrm{Ph} \curvearrowright \mathrm{O}^{+} \mathrm{NC} \widehat{\mathrm{CN}} \underset{\text { cyclohexane, } 40^{\circ} \mathrm{C}, 2 \mathrm{~h}}{\stackrel{\mathrm{Er}(\mathrm{btc})(\mathrm{ED})_{0.75}\left(\mathrm{H}_{2} \mathrm{O}\right)_{0.25}}{\longrightarrow}} \underset{\mathrm{H}}{\mathrm{Ph}}\right\rangle_{\mathrm{CN}}^{\mathrm{CN}}
$$

2005 年 Lin 课题组 ${ }^{[69]}$ 用具有手性的(R)-L3 作为配 体，以 $\mathrm{Ti}\left(\mathrm{O}^{i} \mathrm{Pr}\right)_{4}$ 金属节点供体，制备了具有手性的 Ti-MOF 金属催化剂. 研究发现, 该催化剂能高效的催 化有机锌试剂与醛的加成反应，生成具有手性结构的 醇, 其 ee 值相对较高, 在 80\%以上(Scheme 14).

MOFs 材料不仅可以直接作为催化剂使用, 还可以 作为配体制备相应的过渡金属催化剂. 2015 年 Lin 课题 组 ${ }^{[70]}$ 用同样具有手性的联萗二苯磷(BINAP)作为配体, 以 $\mathrm{ZrCl}_{4}$ 为节点金属, 三氟乙酸 (TFA) 为添加剂, 在 $\mathrm{DMF}$ 中制备了 $\mathrm{Zr}(\mathrm{BINAP})-\mathrm{MOF}(\mathbf{I})$, 再加入 $\mathrm{Rh}(\mathrm{nbd})_{2} \mathrm{BF}_{4}(\mathrm{nbd}$ 为降冰片二烯), 在一定条件下制备了以 $\mathbf{I}$ 为配体的金属 催化剂 $\mathrm{Rh}\left(\mathbf{I} \cdot \mathrm{BF}_{4}\right)$-MOF 催化剂, $\mathrm{Rh}\left(\mathrm{I} \cdot \mathrm{BF}_{4}\right)-\mathrm{MOF}$ 同样具 


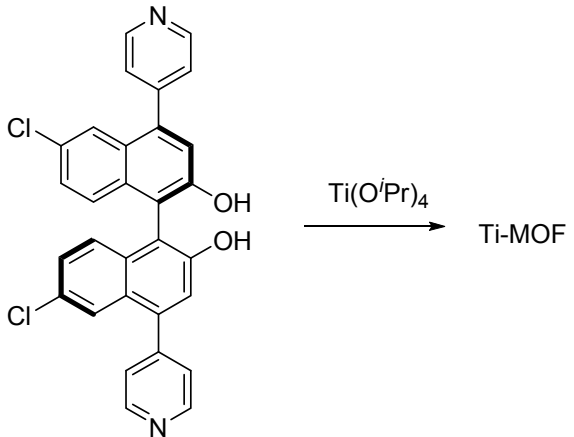

$(R)$-L3

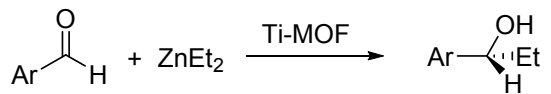<smiles>OCc1ccccc1</smiles>

$e e=88 \%$<smiles>OCc1ccc(Cl)cc1</smiles>

$e e=86 \%$

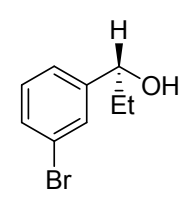

$e e=84 \%$
图式 14 Ti-MOF 的制备及应用

Scheme 14 Preparation and application of Ti-MOF 有很高的催化活性, 在氢气存在下, 能催化 1,6-烯炔的 不对称还原环化反应(Eq. 30), 生成具有手性的环状化 合物. 研究结果表明取代基不同, 对其 $e e$ 值有较大影 响.

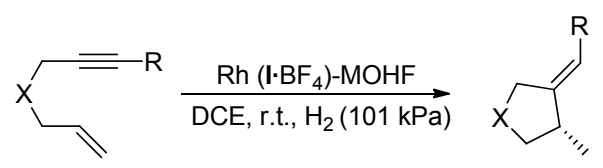<smiles>COc1ccc(C=C2COC[C@H]2C)cc1</smiles><smiles>Cc1ccc(C=C2C[Al]([Al])C[C@H]2C)cc1</smiles><smiles>C[C@@H]1CN([AlH2])CC1=Cc1ccccc1</smiles>

Yield $/ \% \quad 95 \quad 87$ 70 83<smiles>[R]C#CC([Y])[X]</smiles>

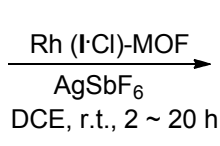<smiles>[R]C=C1C([Y])=CCC1/C=C\CC</smiles><smiles>CC/C=C/C1COC/C1=C\c1ccc(C(F)(F)F)cc1</smiles>

Yield/\% 99 65

99<smiles>CC/C=C/C1CN(Cc2ccccc2)C(=O)/C1=C\c1ccccc1</smiles><smiles>C/C=C/C=C/C1CNC(=O)C1=O</smiles>

99 ee $/ \% \quad 99$

BINAP)，用与制备 I 相同的方法制备了 $\mathrm{Zr}$ (Sub-BINAP)$\mathrm{MOF}(\mathrm{II})$, 再以 II 为配体, 以 $[\mathrm{Rh}(\mathrm{nbd}) \mathrm{Cl}]_{2}$ 为金属供体, 制备了 $\mathrm{Rh}(\mathrm{II} \cdot \mathrm{Cl})-\mathrm{MOF}$ 催化剂. $\mathrm{Rh}(\mathrm{II} \cdot \mathrm{Cl})-\mathrm{MOF}$ 对 $\alpha, \beta$-不 饱和羰基化合物与 1,6-烯炔的不对称还原环化反应有很 好的催化效果(Eq. 32). 反应产率相对较高, 基本上保持 在 $80 \%$ 以上，但产物的立体选择性较低，ee 值在 $10 \%$ $82 \%$ 间.
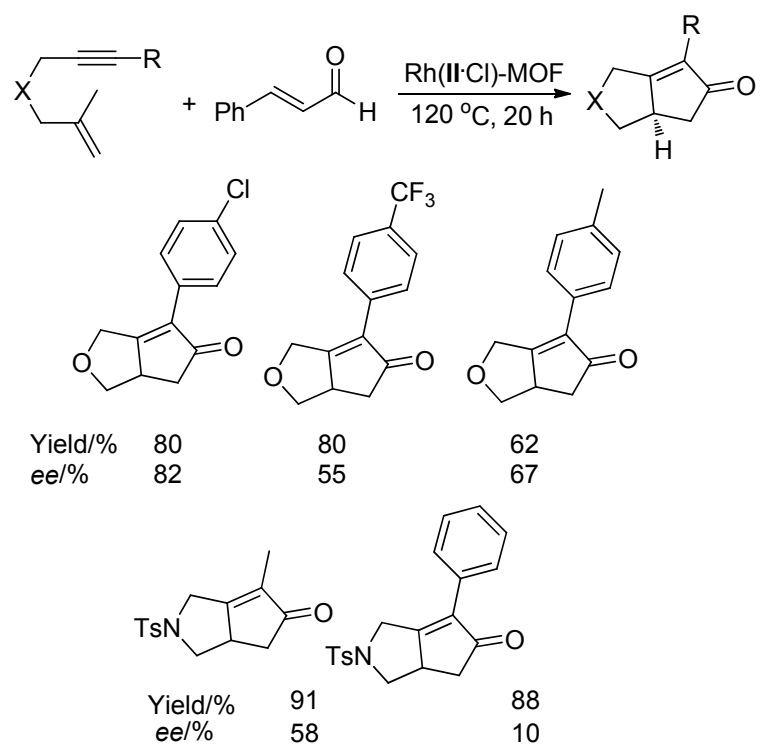

近期 Lin 课题组 ${ }^{[71]}$ 又报道了以 4,4'-二烯丙基-2,2'联吡定(bpyv)和氯化钴作为原料合成的 Co(bpyv)-MOF. 催化性能研究表明 Co(bpyv)-MOF 是烯烃还原反应的高 效催化剂(Eq. 33). 计算发现, 该催化剂中 Co 的负载量 仅为 $0.01 \%$. 该反应的底物适用范围广泛, 不管是环烯 
烃还是链烯烃都能被还原，另外，该催化剂是有选择性 的，只能还原烯烃双键，不能还原苯环双键. 同时，该 课题组还以 $2,2^{\prime}$-联吡啶(bpy)和氯化钴为原料制备了 Co(bpy)-MOF，该材料同样能催化烯烃双键的加氢反应， 但是 $\mathrm{Co}$ 的负载量远大于 $\mathrm{Co}(\mathrm{bpyv})-\mathrm{MOF}$, 为 $0.1 \%$. 采用 类似的方法, 作者还制备了 $\mathrm{Co}(\mathrm{mPT})-\mathrm{MOF}$ (Eq. 34). Co(mPT)-MOF 对烯烃双键的加氢反应的催化效果相对 Co(bpyv)-MOF 较差, 但它能很好地催化硼酸频哪醇在 烯烃双键上的加成反应, 反应条件简单, $23{ }^{\circ} \mathrm{C}$ 下就能完 全反应(注: 虚线为双键所在位置).

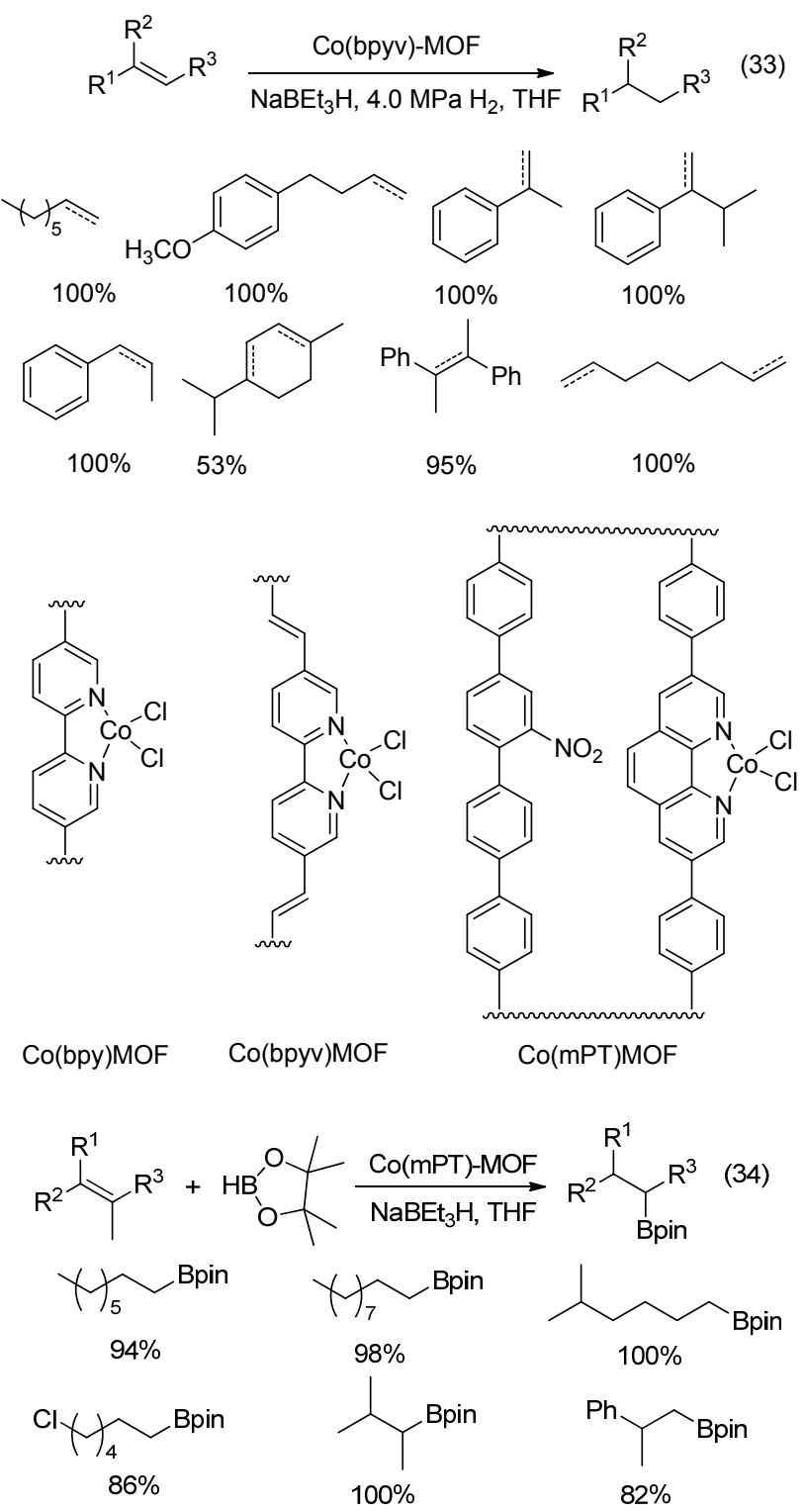

\section{7 总结及展望}

金属有机框架物由于有独特的结构和广泛的应用 前景, 近年来已成为众多科研工作者研究的对象, 尤其 是利用其特殊的框架结构、很大的比表面积和低配位金
属的特点在催化的有机合成反应中被用作多相催化剂. 与传统的均相催化剂相比, MOFs 材料具有相近甚至更 强的催化活性和更好的选择性，催化剂易回收并可以循 环使用多次. 但是, MOFs 作为多相催化剂在有机反应 中的应用也存在着以下几点问题：(1)由于高温会破坏 MOFs 的框架结构，甚至会使其聚合从而影响催化活性， 其所适用的反应温度普遍较低; (2)对 MOF 的研究大多 还是在制备和表征上，对其在有机反应中的作用机理的 研究还不很成熟; (3)现在的 MOFs 大多采用廉价金属 作为金属结点，并且涉及金属种类较少，对贵金属作为 金属结点的 MOFs 研究更少. 因此, 今后 MOFs 的研究 将朝着以下几方面发展; (1)提高 MOFs 对空气、水、高 温的耐受性; (2)扩大构成 MOFs 的金属和有机配体的种 类, 开发兼具配位、活化、链接的多功能有机连接剂, 合 成新型 MOFs，探索其催化应用; (3)合成可协同催化的 双金属甚至多金属 MOFs，探讨其催化机理，扩展 MOFs 在有机反应中的应用范围; (4) MOFs 的纳米化及 纳米 MOFs 在催化与有机合成领域的应用; (5)具有手性 结构的 MOFs 的制备及在手性拆分、不对称合成与催化 反应中的应用等. 我们有理由相信，不久的将来 MOFs 在催化的有机反应研究中将有更佳表现.

\section{References}

[1] Chae, H. K.; Kim, J.; Siberio-Pérez, D. Y.; Eddaoudi, M.; Go, Y.; Matzger, A. J.; O'Keeffe, M.; Yaghi, O. M. Nature 2004, 427, 523.

[2] Jia, C.; Yuan, X.; Ma, Z. Prog. Chem. 2009, 21, 1954 (in Chinese). (贾超, 原鲜霞, 马紫峰, 化学进展, 2009, 21, 1954.)

[3] Song, G.; Wang, Z.; Wang, L.; Li, G.; Huang, M.; Yin, F. Chin. J. Catal. 2014, 35, 185 (in Chinese). (宋国强, 王志清, 王亮, 李国儒, 黄敏建, 银凤翔, 催化学报, 2014, 35, 185.)

[4] Liu, L.; Zhang, X.; Gao, J.; Xu, C. Chin. J. Catal. 2012, 33, 833 (in Chinese).

(刘丽丽，张金金，高金森，徐春明，催化学报, 2012, 33, 833.)

[5] Jiang, Y. J.; Huang, J.; Kasumaj, B.; Jeschke, G.; Hunger, M.; Mallat, T.; Baiker, A. J. Am. Chem. Soc. 2009, 131, 2058.

[6] Li, L.; Lv, Y.; Li, J.; Dong, X.; Gao, S. Prog. Chem. 2012, 24, 747 (in Chinese). (黎林清, 吕迎, 李军, 董晓丽, 高爽, 化学进展, 2012, 24, 747.)

[7] Qiu, W.; Wang, Y.; Li, C.; Zhan, Z.; Zi, X.; Zhang, G.; Wang, R.; He, H. Chin. J. Catal. 2012, 33, 986 (in Chinese) (邱文革, 王昱, 李传强, 展宗城, 訾学红; 张桂臻; 王锐; 何洪, 催化学报, 2012, 33, 986.)

[8] Mondal, S. S.; Holdt, H. R. Angew. Chem., Int. Ed. 2015, 54, 2.

[9] Wang, F.; Yu, R.; Wu, X.; Lu, C. Inorg. Chem. Commun. 2012, 19, 70.

[10] Yang, J.; Liu, Q.; Sun, W. J. Solid State Chem. 2014, 218, 50.

[11] Wu, T.; Zhang, P.; Ma, J.; Fan, H.; Wang, W.; Jiang, T.; Han, B. Chin. J. Catal.. 2013, 34, 167 (in Chinese). (吴天斌，张鹏，马珺，校红雷; 王伟涛; 姜涛, 韩布兴，催化学 报, 2013, 34, 167.)

[12] Callej, G.; Sanz, R.; Orcajo, G.; Briones, D.; Leo, P.; Martínez, F. Catal. Today 2014, 227, 130.

[13] Sierra, M. P.; Almansa, A. M. R.; Corma, A.; Iglesias, M.; Sánchez, 
F. J. Catal. 2013, 299, 137.

[14] Jiang, Y.; Huang, J.; Kasumaj, B.; Jeschke, G.; Hunger, M.; Mallat, T.; Baiker, A. J. Am. Chem. Soc. 2009, 131, 2058.

[15] Kang, X. C,; Liu, H. Z.; Hou, M. Q.; Sun, X. F.; Han, H. L.; Jiang, T.; Zhang, Z. F.; Han, B. X. Angew. Chem., Int. Ed. 2016, 55, 1080 .

[16] Yu, J. C.; Cui, Y. J.; Wu, C. D.; Yang, Y.; Chen, B. L.; Qian, G. D. J. Am. Chem. Soc. 2015, 137, 4026.

[17] Dou, Z. H.; Yu, J. C.; Cui, Y. J.; Yang, Y.; Wang, Z. Y.; Yang, D. R.; Qian, G. D. J. Am. Chem. Soc. 2014, 136, 5527.

[18] Liu, J. W.; Chen, L. F.; Cui, H.; Zhang, J. Y.; Zhang, Li.; Su, C. Y. Chem. Soc. Rev. 2014, 43, 6011.

[19] Zhao, L.; Zeng, H. P. Chin. J. Org. Chem. 2012, 32, 1633 (in Chinese).

(赵莉, 曾和平, 有机化学, 2012,32, 1633.)

[20] Liu, J. W.; Chen, L. F.; Cui, H.; Zhang, J Y.; Zhang, L.; Su, C.-Y. Chem. Soc. Rev. 2014, 43, 6011.

[21] Chughtai, A. H.; Ahmad, N.; Younus, H. A.; Laypkovc, A.; Francis, V. Chem. Soc. Rev. 2015, 44, 6804.

[22] Kumar, R. S.; Kumar, S. S.; Anbu, M. K. Microporous Mesoporous Mater. 2013, 168, 57.

[23] Jiang, D.; Urakawa, A.; Yulikov, M.; Jeschke, G.; Baiker, A. Chem. Eur. J. 2009, 15, 12255.

[24] Jiang, D.; Mallat, T.; Meier, D. M.; Urakawa, A.; Baiker, A. J. Catal. 2010, 270, 26.

[25] Qi, Y.; Luan, Y.; Yu, J.; Peng, X.; Wang, G. Chem. Eur. J. 2015, 21, 1589.

[26] Nguyen, T. T.; Nguyen, K. D.; Phan, N. T. S. Appl. Catal. A: Gen. 2012, 425, 44.

[27] Saggadi, H.; Luart, D.; Thiebault, N.; Estel, L.; Polaert, I.; Len, C. Catal. Commun. 2014, 44, 15.

[28] Nguyen, T. T.; Nguyen, K. D.; Phan, N. T. S.; Vo, A. T. S. Appl. Catal. A: Gen. 2013, 464, 135.

[29] Nguyen, T. T.; Nguyen, K. D.; Phan, N. T. S. Catalysis 2014, 11, 9.

[30] Calleja, G.; Sanz, R.; Orcajo, G.; Briones, D.; Leo, P.; Martínez, F. Catal. Today 2014, 227, 130.

[31] Nguyen, Tung. T.; Nguyen, C. V.; Nguyen, Thao. T.; Phan, N. T. S. Appl. Catal. A: Gen. 2013, 457, 69.

[32] Li, Z.; Xue, L.; Wang, L.; Zhang, S.; Zhao, B. Inorg. Chem. Commun. 2013, 27, 119.

[33] Luz, I.; F. X. Xamena, L.; Corma, A. J. Catal. 2010, 276, 134.

[34] Nguyen, T. T.; Nguyen, K. D.; Phan, N. T. S. Appl. Catal. A: Gen. 2013, 306, 38.

[35] Huang, H.; Arman, H. D.; Li, P.; Regati, S.; Zhao, J.; Chen, B. Chin. Chem. Lett. 2015, 26, 6.

[36] Nguyen, T. T.; Phan, N. T. S. RSC Adv. 2014, 4, 52307.

[37] Nguyen, D. T.; Dang, G. H.; Le, D. T.; Truong, T.; Phan, N. T. S. J. Mol. Catal. A: Chem. 2014, 395, 300.

[38] Truong, T.; Nguyen, V. T.; Phan N. T. S. Catal. Lett. 2014, 144, 1877.

[39] Truong, T.; Dang, G. H.; Tran, N. V.; Truong, N. T.; Le, D. T.; Phan, N. T. S. J. Mol. Catal. A: Chem. 2015, 409, 110.

[40] Ho, S. L.; Yoon, I C.; Cho, C. S.; Choi, H. J. J. Organomet. Chem. 2015, 791, 13 .

[41] Shultz, A. M.; Farha, O, K.; Hupp, J. T.; Nguyen, S. T. J. Am. Chem. Soc. 2009, 131, 4204.

[42] Facchin, G.; Bertani, R.; Berton, A. Inorg. Chim. Acta 1988, 94, 165.

[43] Neogi, S.; Sharma, M. K.; Bharadwaj, P. K. J. Mol. Catal. A: Chem. 2009, 299, 1.
[44] Le, K. A.; Phan, T. D.; Phan, N. T. S. Appl. Catal. A: Gen. 2010, $382,246$.

[45] Zhu, W.; He, C.; Wu, X.; Duan, C. Inorg. Chem. Commun. 2014, $39,83$.

[46] Nguyen, T. T.; Ta, A. H.; Phan, N. T. S. J. Mol. Catal. A: Chem. 2012, 365, 95.

[47] Zhang, Z.; Xu, X.; Yang, Y.; Nosheen, F.; Saleem, F.; Wang, X. Angew. Chem. 2014, 126, 1.

[48] (a) Huang, L.; Liu, W.; Wu, J.; Fu, Y.; Wang, K.; Huo, C.; Du, Z. Tetrahedron Lett. 2014, 55, 2312.

(b) Gang, F.; Dong, T; Xu, G.; Fu, Y.; Du, Z. Heterocycles 2015, 91, 1964.

(c) Xu, G.; Zhang, Y.; Wang, K.; Fu, Y.; Du, Z. J. Chem. Res. 2015, $39,399$.

[49] Li, H. L.; Eddaoudi, M.; O'Keeffe, M.; Yaghi, O. M. Nature 1999, 402, 276.

[50] Opelt, S.; Türk, S.; Dietzsch, E.; Henschel, A.; Kaskel, S.; Klemm, E. Catal. Commun. 2008, 9, 1286.

[51] Huang, L.; Wang, H.; Chen, J.; Wang, Z.; Sun, J.; Zhao, D.; Yan, Y. Microporous Mesoporous Mater. 2003, 58, 105.

[52] Gao, S. X.; Zhao, N.; Shu, M. H.; Che, S. N. Appl. Catal. A: Gen. 2010, 388, 196.

[53] Dong, Z.; Le, X.; Liu, Y.; Dong, C.; Ma, J. J. Mater. Chem. A 2014, 2, 18775 .

[54] Chen, L.; Gao, Z.; Li, Y. Catal. Today 2015, 134, 122.

[55] Bloch, E. D.; Britt, D.; Lee, C.; Doonan, C. J.; Uribe-Romo, F. J.; Furukawa, H.; Long, J. R.; Yaghi, O. M. J. Am. Chem. Soc. 2010, 132, 14382.

[56] Rostamnia, S.; Alamgholiloo, H.; Liu, X. J. Colloid Interf. 2016, 469, 310.

[57] Manna, K.; Zhang, T.; Lin, W. B. J. Am. Chem. Soc. 2014, 136, 6566.

[58] Sierra, M. P.; Almansa, A. M. R.; Corm, A.; Marta, I.; Sánchez, F. J. Catal. 2013, 299, 137.

[59] Langmi, H. W.; Mathe, M.; Bessarabov, D.; Ren, J. W. Int. J. Hydrogen Energy 2014, 39, 890.

[60] Herbst, A.; Khutia, A.; Janiak, C. Inorg. Chem. 2014, 53, 7319.

[61] Sels, B. F.; Gascon, J. ChemCatChem 2014, 6, 2211.

[62] Férey, G.; Hwang, Y. K.; Hong, D. Y.; Chang, J. S.; Jhung, S. H.; Seo, Y. K.; Kim, J.; Vimont, A.; Daturi, M.; Serre, G. Angew. Chem., Int. Ed. 2008, 47, 4144.

[63] Saikia, M.; Bhuyan, D.; Saikia, L. New J. Chem. 2015, 39, 64.

[64] Abney, C. W.; Lin, W. B.; Manna, K.; Zhang, T.; Carboni, M. J. Am. Chem. Soc. 2014, 136, 13182.

[65] David K.; Cejka, J. R.; Kikhtyanin, O. Catal. Today 2014, 237, 236.

[66] Thacker, N. C.; Lin, Z. K.; Zhang, T.; Gilhula, J. C.; Abney, C. W.; Lin, W. B. J. Am. Chem. Soc. 2016, 138, 3501.

[67] Glöggler, S.; Bouchard, L. S.; Brown, J. W.; Nguyen, Q. T.; Otto, T.; Jarenwattananon, N. N. Catal. Commun. 2015, 59, 50.

[68] Ren, Y.; Lu, J.; Jiang, O.; Cheng, X.; Chen, J. Chin. J. Catal. 2015, 36, 1949 (in Chinese).

(任颜卫，陆家贤，江鸥，程晓飞，陈俊，催化学报，2015，36, 1949.)

[69] Wu, C. D.; Hu, A. G.; Zhang, L.; Lin, W. B. J. Am. Chem. Soc. 2005, 127, 8940 .

[70] Sawano, T.; Thacker, N. C.; Lin, Z. K.; McIsaac, A. R.; Lin, W. B. J. Am. Chem. Soc. 2015, 137, 12241.

[71] Zhang, T.; Manna, K.; Lin, W. B. J. Am. Chem. Soc. 2016, 138, 3241 . 